\title{
Gestion de l'agrobiodiversité dans un village de Vanua Lava (Vanuatu) : stratégies de sélection et enjeux sociaux
}

Sophie Caillon et Virginie Lanouguère-Bruneau

\section{(2) OpenEdition \\ Journals}

Édition électronique

URL : http://journals.openedition.org/jso/451

DOI : $10.4000 /$ jso. 451

ISSN : $1760-7256$

Éditeur

Société des océanistes

Édition imprimée

Date de publication : 1 décembre 2005

Pagination : 129-148

ISSN : 0300-953x

\section{Référence électronique}

Sophie Caillon et Virginie Lanouguère-Bruneau, « Gestion de l'agrobiodiversité dans un village de Vanua Lava (Vanuatu) : stratégies de sélection et enjeux sociaux », Journal de la Société des Océanistes [En ligne], 120-121 | Année 2005, mis en ligne le 27 novembre 2008, consulté le 19 avril 2019. URL: http://journals.openedition.org/jso/451 ; DOI : 10.4000/jso.451 


\title{
Gestion de l'agrobiodiversité dans un village de Vanua Lava (Vanuatu) : stratégies de sélection et enjeux sociaux
}

\author{
par
}

\author{
Sophie CAILLON* et Virginie LANOUGUÈRE-BRUNEAU**
}

\section{RÉSUMÉ}

Afin de comprendre comment et pourquoi les agriculteurs choisissent leur portefeuille de cultivars de taros, une étude ethnobotanique a été menée sur l'île de Vanua Lava qui se distingue des autres îles Banks par ses tarodières, irriguées en alternance. Dans le village de Vètuboso, le nalot (pudding) préparé à base de taros, dont les meilleurs sont cultivés dans les tarodières, scande tous les moments importants de la vie sociale. Les agriculteurs choisissent leur portefeuille de vingt cultivars parmi les quatre-vingt-seize présents dans le village, distingués selon onze critères morphologiques et nommés principalement en fonction de leur origine (reproduction sexuée, mutation somatique ou introduction). Sur un échantillon de douze agriculteurs, six cultivars qualifiés de communs ( $83 \%$ des pieds de taros) sont choisis pour leurs atouts agronomiques et organoleptiques. À l'inverse, les quarante cultivars rares (8\% des pieds), piliers de la diversité, sont conservés à des fins sociales pour préserver un héritage familial ou une parcelle d'histoire mythique, pour se démarquer des autres et pour valoriser un produit d'échange. Sachant que seule la prise en compte exhaustive des parcelles permet de recenser l'ensemble des cultivars du village, c'est au final la multiplicité des stratégies des "collectionneurs", des " sélectionneurs» et des " conservateurs », qui dessine la richesse du patrimoine cultural du village et l'inscrit dans la logique de diversité culturelle propre aux sociétés mélanésiennes.

MoTS-CLÉS : classification populaire, conservation in situ, Colocasia esculenta, ethnobotanique, irrigation, nomenclature, tarodière.

* Centre IRD-Orléans, sophie.caillon@orleans.ird.fr.

**vlanouguere@freesurf.fr.

\section{ABSTRACT}

An ethnobotanic research was realised to understand how and why farmers are choosing their cultivars' portfolio in a village named Vêtuboso from Vanua Lava island, well-known for its irrigated pondfields. The nalot (pudding) prepared with taros which tastied and firmest ones are cultivated on pondfields is essential to each step of the social life. Farmers are choosing twenty cultivars among the ninety-six planted in the village that are distinguished thanks to eleven morphogical criteria and named according to their origin (sexual reproduction, somatic mutation and introduction). In a sample of twelve farmers, six of them called "common cultivars" ( $83 \%$ of all planted taros) are selected because of their agronomic and organoleptic qualities. At reverse, forty rare cultivars ( $8 \%$ of plants) are conserved for their social value to preserve a familial (heirloom) or mythic heritage, to be different from the other farmers, andlor to hold a valuable exchange product. To conserve the whole village's diversity, all ponds have to be taken into account. Thus, it is the diversity of individual strategies, classified in "collectors", "selectors" and "conservators", that is responsible from the richness of the farming patrimonial, and that comes within the scope of cultural diversity's logic, peculiar to Melanesian societies.

KEYwORDs: folk classification, in situ conservation, Colocasia esculenta, ethnobotanic, irrigation, nomenclature, pondfield. 
Le taro dit « ordinaire » ou localement appelé « taro des îles » (Colocasia esculenta (L.) Schott) de la famille des Araceae est la principale culture vivrière sur la côte ouest de Vanua Lava ${ }^{1}$, une des quatre-vingt-une îles du Vanuatu (Figure 1). Comme le souligne Jacques Barrau (1983 : 14), « les aliments ne sont pas seulement bons à manger, ils sont aussi bons à penser ». Il en est ainsi du taro à Vanua Lava, qui au-delà de son rôle alimentaire et cérémoniel révèle l'identité du groupe qui le cultive. De la même façon qu'André Georges Haudricourt avait parlé de « civilisation de l'igname» (1964: 93) en Nouvelle-Calédonie, nous pouvons qualifier les sociétés de la côte ouest de Vanua Lava de « civilisation du taro ». Enfin, comme l'ont montré Vandana Shiva (1996) et Arturo Escobar (1998), la valorisation de l'agrobiodiversité est garante de l'identité sociale à l'échelle régionale, de la communauté ou de la personne.

Le village de Vētuboso a été choisi pour l'importance de ses tarodières, sachant qu'il est le plus grand village de l'île avec 129 foyers et 610 habitants (Hess ${ }^{2}$, comm. pers.) qui parlent le vurës ${ }^{3}$ que nous utiliserons pour rendre compte des termes locaux ${ }^{4}$. Vētuboso est situé dans les montagnes à une demi-heure de marche de la côte sud-ouest et à trois heures de Sola $(16 \mathrm{~km})$, la capitale administrative de la Province Torba. Les voies de communication étant précaires, les habitants de Vētuboso se procurent leur faible revenu monétaire grâce au copah ${ }^{5}$ et, pour une minorité, à de petites échoppes ; ils ne tirent que très rarement un revenu de la vente de cormes frais de taro auprès des professeurs du village ou sur le marché de Sola.

Après une brève description des trois systèmes de culture par voie d'eau ou « humide » dont les terrasses irriguées, lieux socialement valorisés, permettent la culture d'un grand nombre de cultivars, nous montrerons à l'aide d'outils empruntés à l'agronomie et à l'anthropologie, comment et selon quels critères les agriculteurs de Vētuboso composent leur portefeuille de cultivars ${ }^{6}$

FIGURE 1 : Site d'étude, le village de Vētuboso sur l'île de Vanua Lava, archipel des Banks au nord du Vanuatu (Pacifique Sud).

1. Vanua Lava, la plus grande île du groupe des Banks, couvre $331 \mathrm{~km}^{2}$ et abrite 1933 personnes (Wells, 2000).

2. Sabine Hess réalise son doctorat sous le titre « Perceptions of place and concept of the person on Vanua Lava, Vanuatu » au département d'Anthropologie de l'université nationale australienne (ANU). Elle a effectué un terrain d'étude à Vêtuboso entre 2000 et 2001. Ces données datent de mars 2002.

3. Alors que le vera'a est utilisé dans le village voisin de Vatrata.

4. Les termes en vurës seront écrits en gras et ceux en bichelamar, langue véhiculaire du Vanuatu, en italique. Les noms locaux en vurës sont transcrits selon l'alphabet établi par Catriona Hyslop (1999) et les habitants de Vanua Lava dans un dictionnaire. La phonétique des termes est présentée en annexe.

5. Albumen sec des noix de coco dont est extrait de l'huile.

6. Nous définissons le terme cultivar comme un morphotype ou un groupe d'individus présentant des caractéristiques morphologiques, agronomiques et organoleptiques suffisamment remarquables et semblables pour que les agriculteurs de Vētuboso lui attribuent un nom particulier, reconnu par l'ensemble de la communauté. D'après notre définition, chaque nom local de taro correspond à un morphotype que l'on nommera cultivar dans la suite du texte. Par portefeuille de cultivars, nous entendons l'ensemble du patrimoine variétal qu'un agriculteur cultive au moment de l'enquête. 
en les identifiant, en les sélectionnant et en les multipliant.

\section{Les tarodières de Vanua Lava : des lieux sociale- ment valorisés}

\section{Les cultures du taro dans les tarodières}

Même s'ils cultivent aussi des jardins mixtes en culture pluviale sur un sol relativement riche ${ }^{7}$, les habitants des villages Vētuboso et Vatrata cultivent principalement les taros sur des terrasses irriguées suivant un savoir-faire unique au Vanuatu (Lanouguère-Bruneau, 1999). Trois systèmes de culture humides peuvent y être différenciés (Yen, 1990; Vargo and Ferentinos, 1991 ; Kirch, 1994 ;Spriggs, 1981 ; Kahn, 1984) :

- en bassins ou casiers irrigués $\left(68,5 \%^{8}\right)$ : $q \bar{e} l$, pl. qèlaqè $l$, en langue vurës,

- en inondation simple parmi les pierres des rivières $(27,5 \%)$. Elle est dite globalement mat ce qui signifie "rivière» et regroupe les mat wöwöres «quelque chose de petit et nombreux » lorsque les taros sont plantés parmi des petites pierres et les mat vetvet « pierres » quand le lit de la rivière est tapissé par de grosses pierres,

- dans la boue autour des rivières ou dans des zones marécageuses sans drainage $(4 \%)$ : mat boak ou boak, ce qui signifie « boue ».

Qu'elles soient constituées de bassins irrigués, de cultures en rivières ou en zones marécageuses, les tarodières irriguées, rot, couvre une surface totale de $17,3 \mathrm{ha}^{9}$ et se répartissent en six grands ensembles respectivement dénommées $\bar{O} t^{10}$ (6 ha), Teñtur « endroit pluvieux» $(0,1 \mathrm{ha})$, Nèlum (0,1 ha), Vebal « deux pierres sont en un endroit » $(0,4 \mathrm{ha})$, Rotluō "grande tarodière » (10,6 ha) et Bokrat ( 0,1 ha). De rares plantations en rivières et en marécages sont également aménagées à l'extérieur de ces grands ensembles et n'ont pas été comptabilisées lors de cette étude. Le potentiel de ces tarodières est très important comparé à d'autres zones du Vanuatu où le taro est planté en bassins irrigués : par exemple, à
Elia sur la côte ouest de Santo, les 1,7 ha de tarodières correspondent à $160 \mathrm{~m}^{2}$ par personne (Walter et Tzerikiantz, 1999) contre $284 \mathrm{~m}^{2}$ à Vētuboso. Les tarodières n'étant pas entièrement utilisées, seuls $187 \mathrm{~m}^{2}$ sont plantés par personne soit $679 \mathrm{~m}^{2}$ par famille ${ }^{11}$.

À Vētuboso, des murets et des canaux recouverts par la forêt secondaire indiquent que d'autres tarodières étaient anciennement utilisées. D'après les villageois, les tarodières Teñtur et Nēlum étaient regroupées en une seule nommée $\bar{O} t$, près de laquelle celle de Vetmowor est cultivée par des habitants de Vatrata. Bernard Vienne (1984 : 58-59) a également observé des vestiges de tarodières irriguées qui étaient probablement exploitées sur la côte orientale de l'île par des petites unités familiales dispersées sur les pentes du volcan Suratamatai. Cette déprise est peut-être due à l'importante dépopulation qui a affecté tout l'archipel du Vanuatu avec la venue des Européens.

\section{Les tarodières irriguées: \\ un lieu de compétition sociale}

L'eau, dirigée par des canaux bordés de pierres, est distribuée entre les terrasses étagées suivant les courbes de niveaux sur les versants ou dans les fonds de vallée. Les terrasses sont compartimentées en bassins ou casiers rectangulaires $(q \bar{e} l)$ par des murs de pierres et de terre dont la hauteur est fonction de la pente. Dans les bassins d'une surface moyenne de $87 \mathrm{~m}^{2}$, des diguettes de terre $(10 \mathrm{~cm})$ délimitent des sous-bassins $\operatorname{tin}^{12}$. Un bassin est cultivé par une unité familiale ou une personne. Les murets des bassins et les diguettes des sous-bassins sont agrémentés de plantes utiles, dont certaines sont comestibles alors que d'autres repoussent par leur odeur l'insecte nuisible gōsōs (Papuana spp.), ou encore protègent les taros par leur force magique : cordylines dagare (Cordyline terminalis) et dagaretan̄sar (Cordyline fruticosa), crotons (da) sasqȫn, kirkiar(mamēsin) ou kirkiarqet (Codiaeum variegatum), cycas $\bar{m} \bar{e} l$ (Cycas rumphii), hibiscus waqagal (Hibiscus rosasinensis), bananier wewe-

7. D'après Paul Quantin (1982), les sols de la côte ouest de Vanua Lava sont des andosols insaturés et profonds, formés sur des cendres et des lapillior volcaniques basiques issus du quaternaire. Stables et perméables, ils sont très fertiles grâce à leur capacité à retenir les minéraux et l'eau. Riches en azote et en bases échangeables, ils présentent cependant une faible déficience en phosphore.

8. Les pourcentages de taros issus des bassins irrigués, des rivières et des zones marécageuses ont été calculés sur un échantillon de neuf foyers (Caillon, 2001-2002, observation personnelle).

9. Ces surfaces ont été calculées par Sophie Caillon lors de son terrain à Vētuboso en 2001, grâce à un programme mis au point par François Bonnot (CIRAD-CP) à partir de points GPS relevées autour des tarodières.

10. Certains noms de tarodières n'ont pas été traduits car correspondent à des noms propres.

11. Calculs comptabilisant les cultures de taro en rivières, en zones marécageuses et en terrasses de 9 foyers (S. Caillon, 2001-2002, observation personnelle).

12. Dans les tarodières de Ōt et Rotluō, on peut calculer que 2,1 sous-bassins de $4,7 \mathrm{~m}^{2}$, en moyenne, compartimentent chaque bassin $q \bar{e} l$ (échantillon de 57 bassins, soit 119 sous-bassins). 
seg (Musa sp.), plante chocolat $\bar{m} a l$ (Graptophyllum pictum), aralia dëmël (Polyscias guilfoylei), bagaiou dövulñö (Polyscias scutellaria), évodie seg (Evodia hortensis), nicolaia sör (Horstedtia sp.), herbe-d'Eugène wilewalal (Achyranthes aspera), coléus datamiar (Coleus blumei) et mêvian (Coprosma persicifolia) ${ }^{13}$. Ces plantes, par leurs racines à faible dispersion, permettent de consolider les murets en retenant la terre sans empiéter sur la surface cultivée en taro. Aujourd'hui, certains agriculteurs plantent du chou des îles (sasar, Abelmoschus manihot), du manioc (maniok, Manihot esculenta) ou du kava ( $g \bar{e}$, Piper methysticum) entre les bassins.

Dans les tarodières irriguées des autres îles (aujourd'hui Maewo, Pentecôte et Santo), les taros se développent dans l'eau tout le long de leur cycle de culture, de la plantation à la récolte. L'immersion dans l'eau permet de lutter, entre autres, contre l'insecte Papuana se nourrissant des jeunes cormes. Contrairement à ces pratiques en immersion continue, les agriculteurs de Vētuboso alternent phases de mise en eau et d'assèchement selon les besoins des taros, pour finalement récolter lorsque le sol est sec. Les besoins en eau étant plus réduits, un plus grand nombre de bassins peut être planté. La durée des trois périodes en eau dépend des conditions climatiques, de la qualité du sol et des taros, mais aussi des besoins de l'agriculteur car il peut modifier le temps de maturation des cormes en jouant avec les phases d'assèchement. Lors de sept visites entre juillet 2001 et mars 2002, l'observation de 119 sous-bassins différents nous permettent de quantifier ces alternances : seulement $23 \%{ }^{14}$ des sous-bassins visités étaient immergés. De plus, l'irrigation alternée permet la récolte de taros les plus appréciés car plus fermes que ceux produits en immersion complète et plus savoureux que ceux plantés en zone marécageuse.

Comparée à la culture pluviale, la culture irriguée peut être qualifiée d'intensive et de durable car les taros poussent plus rapidement, les jachères sont plus courtes et les brûlis limités.

Les taros sont propagés de manière végétative par repiquage de boutures composées d'environ $0,5 \mathrm{~cm}$ de corme surmonté de 30 à $50 \mathrm{~cm}$ de vieilles feuilles dont les limbes ont été coupés pour limiter l'évapotranspiration. En rivières et en marécages, l'extrémité du corme est immédiatement replantée entre les pierres ou dans la boue lorsque le taro est récolté. Cette pratique de plantation individuelle à des moments distincts permet une récolte échelonnée tout le long de l'année.

Dans les bassins irrigués, les plantes prélevées lors de chaque récolte sont attachées par leur pétiole au bout d'un bâton nommé gil, et rapportées à la maison. L'agriculteur attend d'avoir une vingtaine de boutures pour les replanter dans un bassin irrigué, que ce soit celui où il les a prélevées ou dans un autre. S'il lui manque des boutures provenant du bassin même, il se les procurera dans un autre de ses bassins ou à défaut dans les jardins de rivière ou de marécage, ou encore il ramassera les taros non plantés poussant spontanément dans les canaux ou sur les murets de terre séparant les bassins dans les tarodières. Ces taros non plantés peuvent être issus de boutures en surnombre rejetées par d'autres agriculteurs, ou de taros ne correspondant pas aux attentes organoleptiques ou agronomiques du planteur $^{15}$. En dernier lieu, il fera appel pour se procurer ses boutures à un réseau d'échange fondé sur les liens de parenté et de mariage.

Dans un même bassin, la récolte est étalée sur un ou deux mois, la plantation des taros se déroulant généralement en trois fois, à peu près toutes les deux semaines. Mais en fait, le rythme de la plantation dépend du climat, de la disponibilité de l'agriculteur et de ses prétentions sociales. Si l'ensoleillement est trop important, l'agriculteur accélérera le rythme de plantation pour éviter que les jeunes taros ne sèchent avant la première mise en eau. Si une famille a besoin d'argent, l'homme privilégiera le travail dans les cocoteraies au détriment de celui des tarodières. Enfin, si un homme décide de conforter son rang social, il plantera beaucoup de taros en une fois pour pouvoir organiser un grand repas communautaire.

Dans les bassins, les taros sont plantés à des densités plus faibles $\left(2,0 \text { pieds } / \mathrm{m}^{2}\right)^{16}$ que celles de la côte ouest de Santo $\left(2,5\right.$ pieds $\left./ \mathrm{m}^{2}\right)$. Les taros y sont récoltés après un assèchement de la tarodière qui dure minimum un mois. Le poids moyen de corme récolté par pied de taro d'un

13. D'autres plantes malak, mē, $\bar{m} e t e r, t \bar{e}$, wesev et wilial n'ont pas été identifiées. L'identification des espèces a été réalisée par Channel Sam (Ministère des Forêts, Port Vila) à partir d'un herbier constitué par S. Caillon.

14. Les mises en eau ne sont pas saisonnières, ce pourcentage est la moyenne des sept moyennes calculées pour chacune des visites.

15. Ces taros sont distincts de ceux au morphotype sauvage distingués par de longs stolons et une vie sauvage, généralement aquatique.

16. Mesures pour Vanua Lava réalisée par S. Caillon en 2001-2002, à partir d'observations menées sur 57 bassins. Pour l'île de Santo, voir Walter et Tzerikiantz (1999). 
bassin irrigué est de $929 \mathrm{~g}^{17}$ de matière fraîche, soit $361 \mathrm{~g}$ de matière sèche ${ }^{18}$. Les rendements sont de 18,3 t/ha de matière fraîche, soit 7,1 t/ha de matière sèche. Ces rendements sont plus faibles que ceux calculés par Spriggs (1982) à Maewo (entre 35,1 à 58,1 t/ha de matière fraîche) et par Walter et Tzerikiantz (1999) à Santo $(31 \mathrm{t} / \mathrm{ha})^{19}$. Ces différences peuvent être imputées à plusieurs facteurs : collecte des échantillons en milieu immergé, présence de résidus végétaux ou organiques lors des mesures, conditions climatiques optimales. Seules des données exprimées en matière sèche permettraient de comparer ces valeurs. Notons cependant que la sécheresse occasionnée par El Niño à partir de mai 2001 serait responsable de la taille des cormes jugée « anormalement» petite par nos informateurs.

Les taros cultivés en rivières aménagées n'ont, en revanche, pas souffert de la sécheresse. Avec des densités ${ }^{20}$ de 3 pieds $/ \mathrm{m}^{2}$, ils pèsent en moyenne $1644 \mathrm{~g}$ de matière fraîche ${ }^{21}(670 \mathrm{~g}$ de matière sèche ${ }^{22}$ ) après avoir été prélevés dans l'eau. Ainsi, les rendements de 49,3 t/ha de matière fraîche $(20,1 \mathrm{t} /$ ha de matière sèche) sont davantage comparables à ceux des bassins continuellement immergés des îles de Maewo et de Santo. La culture en rivière est d'autant plus productive que la croissance des taros y est deux fois plus rapide : elle nécessite 6 à 13 mois dans un bassin irrigué, mais en rivière 4 à 6 mois suffisent. Le rendement des zones marécageuses est intermédiaire, avec 10,2 t/ha de matière fraîche ${ }^{23}$, entre celui des tarodières et des rivières.

Malgré un rendement plus faible, la culture par irrigation alternée reste la plus valorisée à Vanua Lava. Les taros issus des bassins irrigués sont la fierté des hommes de Vētuboso, de ceux " qui savent travailler ${ }^{24}$ ». Un homme qui exploite un grand nombre de terrasses selon les règles ancestrales sera remarqué et admiré pour son travail et pour son savoir-faire. En effet, si aucun entretien n'est nécessaire dans les cultures en rivière et en marécage, les tarodières irriguées demandent un travail constant et des connaissances transmises depuis des générations.
L'apprentissage de toutes ces connaissances débute très tôt ; certains enfants de moins de dix ans plantent des bassins ${ }^{25}$. Ces connaissances recouvrent différents domaines de l'agronomie comme la maintenance des canalisations, le terrassement, la consolidation des murets et le contrôle de l'irrigation. Les tarodières nécessitent un savoir botanique concernant l'usage des plantes éloignant les ravageurs, mais aussi un savoir social qui fait intervenir des pratiques coutumières, des interdits et de la magie.

La qualité des cormes dépend de l'attention de leur cultivateur, car si l'alternance des périodes de mise en eau n'est pas optimale, les cormes peuvent être attaqués par l'insecte Papuana, ou avoir une texture altérée : intérieur mou et extérieur dur (mötöltöl), centre trop ferme (teñurnur) ou gorgé d'eau (görgör $)^{26}$. Le degré de maturité du corme doit être aussi parfaitement reconnu par la simple observation des parties aériennes. Cultivé en rivière, un taro très mature sera plus ferme qu'un jeune alors que si l'on dépasse le stade optimal de maturité d'un taro de bassins, le corme se ramollit. La qualité des cormes dépend également de l'adéquation entre le cultivar choisi, les modes de cultures et les propriétés bio-chimiques du sol que l'agriculteur identifie de manière empirique. Ainsi, la chair d'un même cultivar est plus ferme (qualité recherchée) lorsque le taro est cultivé par irrigation alternée ou en marécage et plus molle lorsqu'il est cultivé en rivière. Même si le corme est ferme, le goût, l'odeur et la couleur brune des taros de marécage sont les moins appréciés.

Les tarodières sont ainsi valorisées par l'importance et la diversité des savoirs nécessaires pour y faire pousser des taros. Partager ses taros avec les autres membres de la communauté donne l'occasion à un homme de faire apprécier et reconnaître son savoir-faire mais les tarodières peuvent aussi être un lieu de compétition sociale. Par exemple, un cultivar nommé sestañ n'est normalement planté qu'entre les pierres de rivière car il a un mauvais rendement en bassins irrigués. Lorsque deux hommes veulent comparer leur savoir, une compétition est organisée : seul

17. Moyenne calculée sur 188 pieds de taros par S. Caillon et K. Malau en Mars 2002 et Juillet-Août 2002. Les pesées ont été effectuées après nettoyage des racines et de leurs résidus de terre.

18. Calcul de matière sèche après séchage à $40^{\circ} \mathrm{C}$ pendant trois jours effectué par $\mathrm{S}$. Caillon sur onze cultivars.

19. Les taros de la côte ouest de Santo pèsent en moyenne $1,25 \mathrm{~kg}$ (Walter et Tzerikiantz, 1999).

20. Mesures sur deux rivières aménagées plantées de 1485 taros.

21. Mesures sur 35 cormes issus de rivières aménagées en mars 2002.

22. Calculs de matière sèche effectués par $\mathrm{S}$. Caillon sur deux cultivars.

23. Avec un corme d'un poids moyen de $1430 \mathrm{~g}$ de matière fraîche, soit $565 \mathrm{~g}$ de matière sèche, et une densité de $1,8 \mathrm{pieds} / \mathrm{m}^{2}$.

24. Selon les termes d'Eli Field Malau, un des chefs coutumiers de Vêtuboso et fieldworker du Centre Culturel de Port Vila (VKS).

25. Ces bassins portent le nom de l'enfant (par exemple « qēlde Brendon »), les taros lui appartiennent (on parlera « des taros de Brendon ») mais ils seront consommés au sein du foyer.

26. La partie du corme consommée est également importante car la base est plus ferme que le haut. 
celui qui connaît les feuilles magiques obtiendra un sestañ de grande taille en bassins.

\section{La culture du taro : une représentation complémentaire des sexes}

La culture du taro est associée à une symbolique sexuelle qui génère certains interdits et certaines prescriptions, dont l'analyse a été réalisée en collaboration avec Sabine Hess ${ }^{27}$.

Ainsi, les femmes ne sont pas autorisées à s'approcher de la source d'eau de la tarodière, de laver leur linge ou de se baigner en amont car leur " vapeur » bune, assècherait les jardins. Ces interdits sont associés au mythe du héros fondateur Lakakerisis ${ }^{28}$; celui-ci vivait dans un endroit dénommé Beut à l'extrémité sud-est de Vanua Lava. Quand ses frères lui dérobèrent sa femme, il décida de quitter Beut et d'entamer sa quête de femmes. A l'époque, les habitants de l'île se nourrissaient d'ignames à bulbilles (wöböw, Dioscorea bulbifera) prélevées dans la forêt. Lakakēris emporta avec lui ce dont il avait besoin (bassins, eau et taros) pour mettre en place des jardins irrigués. Il visita plusieurs endroits et là où les femmes acceptèrent d'avoir des relations sexuelles avec lui, il installa des bassins irrigués. La plus grande surface, regroupant les tarodières de $\bar{O} t$, Vetmowor, Teñtur et Nēlum au-dessus du village de Vētuboso, fut créée par l'union de Lakakēris et $R o$ vōnōlav ${ }^{29}$. L'union sexuelle mythique a donc été indispensable à la mise en place des premiers bassins irrigués. Les autres tarodières Vebal, Rotluō et Bokrat furent aménagées plus tard par les « anciens » au moins trois générations avant les habitants actuels.

Avant l'arrivée des missionnaires, les hommes et les femmes qui travaillaient dans les tarodières étaient nus. Pour assurer une bonne production de taros, la verge des hommes devait toucher le sol afin de le fertiliser : " Si ta verge traîne sur la terre, tu auras à manger $»^{30}$. Leurs testicules devaient toucher l'eau des canaux lorsqu'ils les nettoyaient pour enrichir l'eau d'irrigation: «Tes testicules doivent être mouillées pour que tu puisses manger du taro $»^{31}$. De même, le vagin des femmes devait être ouvert lorsqu'elles s'accroupissaient pour désherber : « Si ton vagin est ouvert vers le sol, tu auras à manger $»^{32}$. Ainsi à Vanua Lava, les organes sexuels masculin et féminin sont nécessaires à la création et la fertilisation des jardins. La terre est comme une femme, fertilisée par l'eau de la rivière, assimilée au sperme des hommes, qui irrigue les bassins. De même, la croissance des taros est similaire à la croissance humaine, et la présence complémentaire de l'homme et de la femme est nécessaire à leur gestation. Une division sexuelle du travail est ainsi instaurée : l'homme s'occupe de créer et d'entretenir les canaux d'irrigation, de planter les boutures avec un bâton à fouir, de gérer les alternances inondation/assèchement dans les bassins, alors que la femme est chargée du désherbage. Dans les faits, le travail dans les tarodières irriguées n'est pas aussi cloisonné : des hommes désherbent et des femmes plantent, ce qui corrobore avec les observations de M. Jolly (1994 : 68) dans l'île de Pentecôte, qui note qu'il n'y a pas de division du travail instituée entre les sexes.

Dans cette île (Jolly : op. cit.), les taros sont perçus comme féminins. À Vanua Lava, les villageois distinguent les taros mâles des taros femelles. Deux trous sculptés dans une pierre, visibles en période sèche, au pied de la tarodière la plus prestigieuse, $\bar{O} t$, représentent cette dualité. Pour toute l'île, les taros mâles viendraient du trou profond et allongé alors que les taros femelles du trou plus petit et plus rond ; la forme respective de ces deux cavités renvoie ainsi à l'image du phallus et du vagin. De fait, les taros dits masculins sont hauts, ont un corme unique allongé et fin, alors que les taros féminins sont plus petits entourés de nombreux rejets. Comme une mère, les taros femelles partagent leur énergie avec leurs enfants. Ainsi, les tarodières irriguées, interprétées comme le produit d'une union sexuelle (la terre féminine fertilisée par l'eau masculine), donnent naissance à des taros mâles et femelles, équivalents de l'humanité composée d'hommes et de femmes.

\section{Une grande diversité de cultivars et d'agriculteurs.}

\section{Les cultivars de Vanua Lava: identification, nomination et description}

Sur la côte ouest de Vanua Lava, 96 cultivars ont été recensés et qualifiés par une description agro-morphologique complétée par des récits d'origine. Cet inventaire a pu être établi à partir d'enquêtes individuelles, de questionnaires (56 interviews rapides et 12 approfondies), d'infor-

\footnotetext{
27. Voir note 2.

28. Seule une version limitée de l'histoire révélée par John-Elizabeth Kökör est livrée ici.

29. Ro est le préfixe féminin ; vōnōlav est le terme en vurës pour désigner l'île Vanua Lava, signifiant « grand lieu habité ».

30. Na qarañ ni rev la tan nek i gen sinag

31. Na loson̄ nin öw nek $i$ gen o qiat

32. Na veseñ ni wawan̄ nek i gengen sinag
} 
mations recueillies au cours de multiples discussions informelles avec les habitants de Vētuboso, Vatrata et Kerepeta, ainsi que d'observations de tarodières. Tous les cultivars nommés sur la côte ouest de Vanua Lava sont plantés à Vētuboso, ce qui n'est pas le cas dans un village voisin, Vatrata, également réputé pour ses tarodières irriguées ${ }^{33}$. Cette différence s'explique, en partie, par la densité de la population et donc des surfaces plantées ; Vatrata n'abrite que 188 personnes, contre 610 habitants à Vētuboso (S. Hess, 2002, communication personnelle).

Les habitants de l'île différencient leurs taros à l'aide de onze critères en distinguant, dans un premier temps, les couleurs et marbrures sur la partie centrale du pétiole et aux deux extrémités, les jonctions avec la feuille et le corme. La couleur, l'intensité, la forme des veines sous les feuilles et à leurs surfaces, la jonction des veines à l'intersection avec le point d'attache du pétiole (mēte ou « œil ») aident l'agriculteur à identifier les cultivars. Il observe ensuite le port général, c'est-à-dire le nombre de rejets (miarēsul ou « beaucoup d'enfants »), de stolons (rev) et la hauteur de la plante. Si un doute subsiste, les agriculteurs grattent le corme pour voir la couleur de la peau puis de la chair. Plus rarement, il se base sur la forme du corme : allongé et long pour les taros mâles, rond et court pour les taros femelles.

Lorsqu'un agriculteur découvre un variant ou un nouveau morphotype, il le repère pour évaluer ses qualités, puis juge de sa place dans son patrimoine pour enfin le rejeter ou le sélectionner. À Vētuboso, cet événement étant rare, l'agriculteur décide toujours de le conserver. Avant de le nommer, il s'assurera qu'il est bien différent des cultivars déjà connus en le montrant aux personnes réputées pour leur savoir. Il le multipliera à l'aide des rejets au cours de plusieurs cycles de culture pour enfin le diffuser ou se le réserver. Dans tous les cas, le "découvreur» s'appropriera le variant, objet nouveau qui lui permettra de se démarquer des autres agriculteurs et d'en tirer du prestige et une certaine fierté.

Comme à Gaua, à Mota (B. Vienne, 2003, communication personnelle) ou à Mota Lava (V. Lanouguère-Bruneau, 1997-1999, observation personnelle), les habitants de Vanua Lava mémorisent soigneusement les récits d'origine qui entourent l'apparition de chaque cultivar. Le contexte de «l'apparition» de chacun des 96 noms de taro a été demandé à quatre chefs de
Vētuboso réputés pour leurs connaissances. Nous avons ainsi recueilli 82 récits (le contexte, le découvreur et/ou le lieu d'apparition) correspondant chacun à un cultivar de taro. Dans le cas de 40 cultivars, cette apparition est récente (depuis les années 1940) et peut être datée précisément. Dans le cas de 30 cultivars, elle est trop ancienne pour être exactement située dans le temps. L'origine des 12 autres cultivars est liée à un mythe fondateur.

Parmi ces 82 cultivars dont l'histoire a été recueillie, 57 cultivars ont pu être classés suivant trois catégories socio-biologiques définie selon leur condition d'apparition. Les autres cultivars ont une histoire d'origine soit controversée, soit mythique. Parmi les 57 cultivars historiquement identifiables, les plus nombreux, soit 27 d'entre eux (47\%), sont apparus après qu'un agriculteur ait défriché par brûlis un bassin en jachère depuis au moins cinq ans, le plus souvent depuis plusieurs dizaines d'années. D'après la description de la jeune plantule et des conditions d'émergence, ces cultivars sont vraisemblablement issus de graines résultant d'autofécondation ou de fécondation croisée (Caillon et al., 2005). Le cultivateur s'appropriera sa découverte en nommant ce nouveau cultivar avec son propre nom précédé de qiat min « taro de » ou wot min « né sous ». Ces préfixes ont tendance à disparaître au cours des années. Les noms des cultivars désignent également les lieux ou les conditions dans lesquelles ils ont été trouvés. Par exemple, le cultivar teweswër, dont le nom est composé des termes tewes "fleur» et wër « hibiscus»(Hibiscus tiliaceus), a été nommé ainsi car il est apparu au sein d'un monticule de fleurs d'hibiscus ramassé à la base de l'arbre par un agriculteur.

Ensuite, $37 \%$ des cultivars (21 d'entre eux) viennent de l'extérieur de l'île : dix ont été acquis dans les îles Banks (Ureparapara, Gaua et Mere Lava), neuf dans les autres îles du Vanuatu (Santo et Maewo) et deux viennent d'un pays étranger (Fidji et les îles Norfolk). Les cultivars ramenés d'une île du groupe des Banks conserveront leur nom vernaculaire après un ajustement selon les correspondances dans la langue de Vētuboso ${ }^{34}$. Les autres, importés d'un lieu plus lointain, porteront le nom de l'île d'origine.

Enfin, suite à une ou des mutations somatiques aléatoires lors de la replantation d'un cultivar connu, l'agriculteur attribuera un autre nom à ce nouveau cultivar distingué par de nouvelles

33. Même si la langue de Vatrata, le vera'a, est différente, les noms des cultivars peuvent être facilement rapprochés de leurs homologues de Vêtuboso en langue vurës.

34. L'homogénéité des langues des îles Banks consiste en une transformation de certaines lettres qui rendent très facile la conversion d'un mot d'une île à l'autre. 
caractéristiques morphologiques, comme la couleur des pétioles. Les villageois reconnaissent ainsi neuf cultivars mutants, soit $16 \%$. Les mutants, prendront le nom du cultivar dont ils sont issus, suivis d'un adjectif reflétant les modifications de couleur : lamkör " foncé » lorsque le pétiole prend des tons plus sombres, qagqag « clair» (où qag signifie «blanc») dans le cas inverse. Quand le corme se strie de lignes (fibres) d'une couleur distincte du reste du parenchyme, les villageois ajoutent le terme gatgat " rayé ", mais lorsqu'il ne s'agit que de points de couleur, lörlör " moucheté » est ajouté. Pour simplement indiquer un changement, malmale $i$ « faux » précède le nom du cultivar originel.

Deux cultivars ramenés de la même île peuvent, d'après le système de nomenclature de Vētuboso, porter le même éponyme de l'île. Cependant les cultivars sont distingués morphologiquement suivant les onze critères locaux décrits ci-dessus ; l'homonymie est donc consciente et connue de tous. Plusieurs processus de sélection peuvent avoir lieu pour ces cultivars homonymes : soit un seul des cultivars portant le même nom sera conservé, soit certains perdront leur identité de cultivar étranger en empruntant, entre autres, le nom en langue vernaculaire d'un animal ou d'une plante dont les caractéristiques morphologiques lui ressemblent (par ex. : la couleur rouge des écrevisses). Le seul cas d'homonymie concerne les taros au morphotype sauvage qui ne sont pas plantés, qui se multiplient dans les rivières ou les canaux d'irrigation grâce à leurs nombreux stolons, et dont les cormes sont petits et irritants. Sous un nom, qiatrev (qiat « taro » et rev "stolon ou qui marche"), les agriculteurs regroupent l'ensemble de ces morphotypes arborant des couleurs et des formes variées.

À l'inverse, cinq cultivars sont identifiés par l'ensemble des habitants par deux noms. Ce doublement de noms reflète une origine incertaine ou une fluidité des règles de nomenclature. Dans le premier cas, le cultivar peut ainsi porter les noms des deux hommes potentiellement responsables de la découverte. Dans le deuxième, le cultivar trouvé a pu être nommé suivant le nom de l'agriculteur qui l'a repéré et le nom du lieu où il est apparu.

Il peut également y avoir des confusions sur la réelle origine du cultivar. Par exemple le cultivar Tanna est un taro acheté sur le marché de Luganville de l'île d'Espiritu Santo, alors qu'un autre cultivar de Tanna avait déjà été apporté quelques mois auparavant à Vētuboso mais n'avait pas survécu. Les villageois n'ont pas différencié l'origine de ces deux cultivars, pourtant distincts morphologiquement, leur attribuant un même nom malgré le discours de l'homme responsable de sa dispersion.

Sur les 96 cultivars plantés à Vētuboso, un cas d'homonymie et cinq de synonymies connus de tous ont été relevés. À part ces exceptions on peut conclure qu'à un nom correspond un cultivar dont les individus sont morphologiquement, gustativement (S. Caillon, 2001-2003, observation personnelle) et génétiquement (Caillon et al., 2005) semblables. Les critères locaux d'identification sont suffisamment fins pour éviter toute confusion.

Une fois que le cultivar a été identifié et nommé, il est classé suivant son état cultivé ou pas, son origine (trouvé, changé, introduit), ses similarités morpho-agronomiques et ses capacités d'adaptation agronomique. Les taros cultivés, turgi qiat "vrais taros », s'opposent aux taros sauvages et ornementaux, non plantés et/ou non consommables, malēe qiat «faux taro ». La famille des taros au morphotype sauvage font partis des faux taros.

Les variétés cultivées ou les cultivars peuvent être associés sous un nom de groupe ou famille, soit parce qu'ils ont la même origine, soit parce qu'ils présentent les mêmes adaptations agronomiques ou des caractéristiques morphologiques similaires. Par exemple, la famille wakata regroupe les cultivars ramenés de Gaua, l'île voisine du Sud, sur une pirogue de ce nom. Dans la famille Marē, tous les cultivars ont des cormes particulièrement allongés et poussent relativement plus vite que les autres cultivars. Enfin même si $90 \%$ des cultivars poussent dans toutes les conditions agro-écologiques, certains cultivars sont conservés pour leur performance dans un agrosystème spécifique sans pour autant être regroupés sous un terme générique. Rēsim, ses-

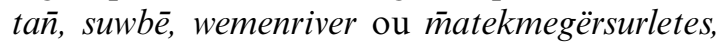
wotanaval et wotkērēvor ne sont plantés qu'en rivières, wegeretqon, wēbigqō et wēbigq $\bar{o}$ mamē qu'en marécages et rov uniquement en bassins irrigués.

Le village de Vētuboso abrite ainsi un grand nombre de cultivars sélectionnés, multipliés et conservés par des agriculteurs ayant des pratiques très différentes.

\section{Les agriculteurs et leur stratégie de gestion de la} diversité.

Après avoir étudié la diversité à l'échelle du village, nous nous sommes intéressés aux stratégies individuelles de gestion (sélection / conservation) de cette diversité. Pour ce faire, neuf foyers d'agriculteurs représentatifs du village ont été choisis pour analyser la distribution des cul- 
tivars entre les bassins et entre les agriculteurs. Un des foyers, étudié plus précisément, comptait trois agriculteurs, amenant à douze le nombre d'agriculteurs interrogés.

Achille $^{35}$, le plus âgé (70 ans) n'a plus d'enfants à charge et continue avec sa femme à entretenir ses jardins. Il n'est pas un homme de savoir mais est respecté pour son grand âge. Vient ensuite Luc (55 ans), qui par son ancien travail d'assistant technique du Service de l'Agriculture $^{36}$, a vécu l'essentiel de son temps dans d'autres îles du Vanuatu. Aujourd'hui retraité, il a acheté un terrain qu'il a transformé en cocoteraie, son père ayant distribué toute ses terres à ses frères qui n'avaient pas quitté le village. Il a aujourd'hui l'une des plus grandes plantations villageoises de la côte ouest (6 ha). Eric et Henri, tous les deux âgés de 45 ans, ont tous deux vécu sur la grande île d'Espiritu Santo au temps du Condominium. De retour dans leur village, vers l'âge de trente ans, ils sont devenus des chefs coutumiers. Certains de leurs enfants sont déjà mariés, d'autres travaillent dans leurs jardins ou sont encore en bas âge. Martin, Anatole et Pascal $(30,27$ et 20 ans) sont des jeunes pères de famille dont les enfants ne sont pas en âge de travailler. Wendy, 22 ans, s'est mariée dans un village nommé Wasaga à $1 \mathrm{H} 30$ de marche, où les taros ne sont pas cultivés car la terre est trop sèche. Quentin, Hervé, Arthur et Bertrand (21, 18, 16 et 8 ans) sont célibataires et aident leur famille en les approvisionnant en cormes. Pascal, Hervé, Arthur, Wendy et Bertrand sont les enfants d'Eric. Les trois plus jeunes, Hervé, Arthur et Bertrand font toujours partie du foyer parental et leurs bassins sont groupés dans l'analyse avec celle de leur père, sous le terme "Famille d'Eric ».

Cinquante et un des 96 cultivars de la côte ouest de Vanua Lava ont été recensés dans les bassins irrigués de notre échantillon (Tableau 1). Parmi eux, six cultivars dits 'communs' - dans l'ordre, rov, marēwasalav, lantar, wasanto, vinmötöl et wêve — représentent à eux seuls $83 \%$ des plants de taros cultivés par les 12 agriculteurs de l'échantillon et au minimum $5 \%$ de chaque portefeuille (Tableau 2). A l'opposé, 40 cultivars, que l'on dénommera " rares ", représentent seulement $8 \%$ de l'ensemble des taros et moins de $1 \% \mathrm{du}$ portefeuille de chaque agriculteur ${ }^{37}$. On compte 7129 pieds pour les six cultivars communs et 662 pieds pour les 40 cultivars rares, soit près de onze fois plus de pieds de cultivars communs, pour sept fois moins de cultivars. Si les villageois possèdent en général les mêmes cultivars communs, les portefeuilles de cultivars rares sont beaucoup plus hétéroclites favorisant ainsi l'exclusivité et valorisant la différence.

Pour estimer la capacité de chaque agriculteur à choisir et garder cette collection de cultivars, les stratégies de sélection et de conservation ont été étudiées pour ce groupe de 12 agriculteurs. Nous avons observé plusieurs indicateurs: les surfaces plantées, le nombre de pieds, la richesse ou le nombre de cultivars et l'indice ShannonWiener. Celui-ci, noté $\mathrm{H}$ dans le tableau 3, est un indicateur de diversité utilisé notamment en écologie (Krebs, 1994). Il se calcule à partir du nombre de cultivars et de leur abondance relative selon la formule :

$$
\mathrm{H}=-\sum_{i=1}^{i=S}(\mathrm{pi}) \log _{2}(\mathrm{pi})
$$

où, pour chaque agriculteur, $\mathrm{S}$ est le nombre total de cultivars qu'il cultive et $\mathrm{p}_{\mathrm{i}}$ est la proportion du ième cultivar dans l'ensemble de ses bassins. Elle permet de répondre à la question « Avec quelle difficulté pourrait-on prédire correctement le nom du cultivar du prochain individu collecté ? ", sachant que plus la difficulté est grande, plus la prédiction du nom est incertaine, plus la diversité sera importante. Dans notre cas, l'indice $\mathrm{H}$ reflète la manière dont l'agriculteur multiplie chacun de ses cultivars. Afin de pouvoir comparer la diversité des 12 portefeuilles de taro, nous utiliserons l'équitabilité ou $\mathrm{E}=\mathrm{H} / \mathrm{H}_{\max }$ avec $\mathrm{H}_{\max }=\log _{2} \mathrm{~S}$. Plus E est élevé, plus la proportion relative de chaque cultivar est identique et donc plus le risque de perte de matériel est limité.

Comme le montre le tableau 3 , chaque agriculteur plante en moyenne $479 \mathrm{~m}^{2}$ de bassins, Bertrand disposant de la surface la plus petite avec ses $20 \mathrm{~m}^{2}$ (rappelons qu'il n'a que 8 ans) et Henri la plus grande avec $1910 \mathrm{~m}^{2}$. Le nombre de pieds plantés varie entre 34 et 2652 (respectivement plantés par Bertrand et Henri) avec une moyenne de 956,7 pieds par cultivateur. Le nombre moyen de cultivars est de 19,7, la médiane est de 14 et l'écart-type de 10,8. Il existe donc une forte variabilité concernant la richesse des portefeuilles, ceux de Bertrand ( 6 cultivars) et d'Henri (46) étant aux deux extrémités de cette distribution. Les nombres moyens de pieds par bassin $(153,7)$ et de cultivars $(9,6)$ sont également variables dans les 56 bassins considérés (écart-type respectif de 133,4 et 4,9 ).

35. Afin de respecter l'anonymat des personnes interrogées, les noms des agriculteurs ont été modifiés.

36. Agent chargé par le Ministère de l'Agriculture de conseiller les villageois sur les nouvelles techniques agricoles.

37. Six cultivars, entre les communs et les rares, sont dits « intermédiaires ». 


\begin{tabular}{|c|c|c|c|c|c|c|c|c|c|c|c|c|}
\hline & Cultivars & $\begin{array}{l}\text { Famille } \\
\text { Eric }\end{array}$ & Pascal & Wendy & Henri & Achille & Luc & Anatole & Martin & Quentin & $\begin{array}{l}\mathrm{Nb} \text { total } \\
\text { pieds / } \\
\text { cultivar }\end{array}$ & $\begin{array}{c}\mathrm{Nb} \\
\text { foyers / } \\
\text { cultivar }\end{array}$ \\
\hline \multirow{6}{*}{ 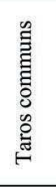 } & Rov & 615 & 69 & 5 & 599 & 115 & 0 & 4 & 617 & 46 & 2070 & 8 \\
\hline & Marēwasalav & 574 & 106 & 19 & 721 & 11 & 13 & 48 & 39 & 120 & 1651 & 9 \\
\hline & Lantar & 377 & 59 & 10 & 570 & 42 & 18 & 69 & 75 & 78 & 1298 & 9 \\
\hline & Wasanto & 22 & 6 & 0 & 13 & 1 & 79 & 42 & 701 & 2 & 866 & 8 \\
\hline & Vinmötöl & 223 & 117 & 10 & 228 & 7 & 9 & 39 & 29 & 65 & 727 & 9 \\
\hline & Wēvē & 156 & 42 & 2 & 76 & 23 & 0 & 4 & 198 & 16 & 517 & 8 \\
\hline \multirow{5}{*}{ 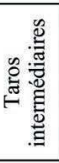 } & Lōkreg & 152 & 20 & 0 & 20 & 16 & 9 & 15 & 33 & 31 & 296 & 8 \\
\hline & Biliag & 0 & 0 & 0 & 163 & 0 & 0 & 0 & 0 & 0 & 163 & 1 \\
\hline & Wakata & 47 & 19 & 0 & 33 & 4 & 1 & 17 & 0 & 13 & 134 & 7 \\
\hline & (Re)Lenman & 74 & 11 & 4 & 21 & 1 & 0 & 0 & 0 & 8 & 119 & 6 \\
\hline & Wederebiliag & 54 & 5 & 21 & 1 & 1 & 0 & 0 & 24 & 1 & 107 & 7 \\
\hline \multirow{42}{*}{ 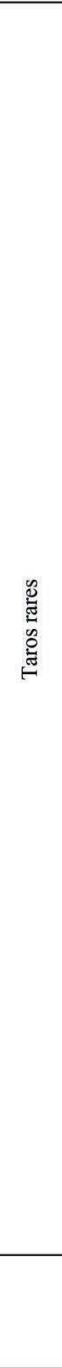 } & Wotlēqitēgidavaqal & 5 & 0 & 0 & 12 & 19 & 1 & 10 & 0 & 35 & 82 & 6 \\
\hline & Varvarsöm & 26 & 0 & 0 & 10 & 10 & 5 & 3 & 0 & 16 & 70 & 6 \\
\hline & (Wotmin)Wērirtel & 5 & 0 & 0 & 10 & 22 & 15 & 0 & 1 & 7 & 60 & 6 \\
\hline & \begin{tabular}{|l|} 
Teweswër \\
\end{tabular} & 14 & 20 & 0 & 4 & 12 & 0 & 0 & 0 & 0 & 50 & 4 \\
\hline & Taltal & 21 & 0 & 0 & 8 & 10 & 1 & 2 & 0 & 0 & 42 & 5 \\
\hline & Wegeretqon & 40 & 0 & 0 & 0 & 0 & 0 & 0 & 0 & 0 & 40 & 1 \\
\hline & Qiatlēlē or Qiatminwog & 33 & 0 & 0 & 3 & 0 & 0 & 0 & 1 & 1 & 38 & 4 \\
\hline & Nalumlum & 0 & 0 & 0 & 24 & 0 & 0 & 0 & 0 & 0 & 24 & 1 \\
\hline & Tanna & 5 & 0 & 0 & 10 & 0 & 0 & 0 & 0 & 2 & 17 & 3 \\
\hline & (We)menriver & 2 & 0 & 0 & 2 & 3 & 3 & 0 & 0 & 6 & 16 & 5 \\
\hline & Mesmamē & 0 & 0 & 0 & 15 & 0 & 0 & 0 & 0 & 0 & 15 & 1 \\
\hline & Wotliev & 0 & 0 & 0 & 1 & 1 & 8 & 3 & 0 & 2 & 15 & 5 \\
\hline & M̄ōvōl & 0 & 0 & 0 & 14 & 0 & 0 & 0 & 0 & 0 & 14 & 1 \\
\hline & Wasē & 0 & 0 & 0 & 14 & 0 & 0 & 0 & 0 & 0 & 14 & 1 \\
\hline & Wamal & 4 & 2 & 2 & 3 & 1 & 0 & 0 & 0 & 1 & 13 & 6 \\
\hline & Rēsim & 0 & 0 & 0 & 0 & 1 & 1 & 0 & 0 & 11 & 13 & 3 \\
\hline & Lantar lamkör & 0 & 0 & 0 & 11 & 0 & 0 & 0 & 0 & 0 & 11 & 1 \\
\hline & (We)M̄ēlēglêg & 7 & 0 & 0 & 3 & 0 & 0 & 1 & 0 & 0 & 11 & 3 \\
\hline & Master & 0 & 0 & 0 & 0 & 10 & 0 & 0 & 0 & 0 & 10 & 1 \\
\hline & Siritimiat & 5 & 2 & 0 & 2 & 1 & 0 & 0 & 0 & 0 & 10 & 4 \\
\hline & Rēlègtêl & 0 & 0 & 8 & 1 & 0 & 0 & 0 & 0 & 0 & 9 & 2 \\
\hline & Burmatan & 0 & 0 & 0 & 8 & 0 & 0 & 0 & 0 & 0 & 8 & 1 \\
\hline & Qiatgōl & 5 & 0 & 0 & 1 & 0 & 0 & 2 & 0 & 0 & 8 & 3 \\
\hline & (Bus)Ōr & 3 & 0 & 0 & 4 & 0 & 0 & 0 & 0 & 1 & 8 & 3 \\
\hline & Agricaltcha & 7 & 0 & 0 & 0 & 0 & 0 & 0 & 0 & 0 & 7 & 1 \\
\hline & Bulalef & 0 & 0 & 0 & 7 & 0 & 0 & 0 & 0 & 0 & 7 & 1 \\
\hline & M̄ēvinvian & 1 & 3 & 0 & 1 & 0 & 0 & 0 & 0 & 2 & 7 & 4 \\
\hline & Römöwuler & 0 & 0 & 0 & 6 & 0 & 0 & 0 & 0 & 0 & 6 & 1 \\
\hline & Wakataqagqag & 0 & 0 & 0 & 5 & 0 & 0 & 0 & 0 & 0 & 5 & 1 \\
\hline & Wotmēlēv & 0 & 0 & 0 & 5 & 0 & 0 & 0 & 0 & 0 & 5 & 1 \\
\hline & Wēbigqō & 0 & 0 & 0 & 4 & 0 & 0 & 0 & 0 & 1 & 5 & 2 \\
\hline & M̄ew & 2 & 0 & 0 & 2 & 0 & 0 & 0 & 0 & 0 & 4 & 2 \\
\hline & (Re)Mesvōlvōl & 0 & 0 & 0 & 4 & 0 & 0 & 0 & 0 & 0 & 4 & 1 \\
\hline & Tan̄evsōs & 0 & 0 & 0 & 4 & 0 & 0 & 0 & 0 & 0 & 4 & 1 \\
\hline & Teñtur or Qiatmingala & 0 & 0 & 0 & 3 & 0 & 0 & 0 & 0 & 0 & 3 & 1 \\
\hline & Novok & 0 & 0 & 0 & 2 & 0 & 0 & 0 & 0 & 0 & 2 & 1 \\
\hline & Rēgēt & 0 & 0 & 0 & 2 & 0 & 0 & 0 & 0 & 0 & 2 & 1 \\
\hline & Orbarbar & 0 & 0 & 0 & 1 & 0 & 0 & 0 & 0 & 0 & 1 & 1 \\
\hline & Siagētgēt & 0 & 0 & 0 & 1 & 0 & 0 & 0 & 0 & 0 & 1 & 1 \\
\hline & Wasanto mamē & 0 & 0 & 0 & 0 & 1 & 0 & 0 & 0 & 0 & 1 & 1 \\
\hline & $\begin{array}{l}\begin{array}{l}\text { Nb total de pieds / } \\
\text { foyer }\end{array} \\
\end{array}$ & 2479 & 481 & 81 & 2652 & 312 & 163 & 259 & 1718 & 465 & 8610 & 9 \\
\hline & $\begin{array}{l}\text { Nb de cultivars / } \\
\text { foyer }\end{array}$ & 27 & 14 & 9 & 46 & 22 & 13 & 14 & 10 & 22 & 51 & \\
\hline
\end{tabular}

TABLeau 1. - Description des taros communs, intermédiaires et rares parmi les 56 bassins plantés par 9 foyers individuels de producteurs. 


\begin{tabular}{|c|c|c|c|c|c|c|}
\hline & \multicolumn{3}{|c|}{ Nombre de pieds (\%) } & \multicolumn{3}{|c|}{ Nombre de cultivars (\%) } \\
\hline & $\begin{array}{c}\text { Taros } \\
\text { communs }\end{array}$ & $\begin{array}{c}\text { Taros } \\
\text { intermédiaires }\end{array}$ & Taros rares & $\begin{array}{c}\text { Taros } \\
\text { communs }\end{array}$ & $\begin{array}{c}\text { Taros } \\
\text { intermédiaires }\end{array}$ & Taros rares \\
\hline Portefeuille Eric & $1207(16,9 \%)$ & $177(21,6 \%)$ & $64(9,7 \%)$ & 6 & 4 & 10 \\
\hline Portefeuille Arthur & $560(7,9 \%)$ & $124(15,1 \%)$ & $121(18,3 \%)$ & 6 & 4 & 17 \\
\hline Portefeuille Hervé & $173(2,4 \%)$ & $19(2,3 \%)$ & $0(0,0 \%)$ & 6 & 3 & 0 \\
\hline Portefeuille Bertrand & $27(0,4 \%)$ & $7(0,9 \%)$ & $0(0,0 \%)$ & 4 & 2 & 0 \\
\hline Portefeuille Famille Eric & $1967(27,6 \%)$ & $327(39,9 \%)$ & $185(27,9 \%)$ & 6 & 4 & 17 \\
\hline Portefeuille Pascal & $399(5,6 \%)$ & $125(6,7 \%)$ & $27(4,1 \%)$ & 6 & 4 & 4 \\
\hline Portefeuille Wendy & $46(0,6 \%)$ & $25(3,1 \%)$ & $10(1,5 \%)$ & 5 & 2 & 2 \\
\hline Portefeuille Henri & $2207(31,0 \%)$ & $238(29,1 \%)$ & $207(31,3 \%)$ & 6 & 5 & 35 \\
\hline Portefeuille Achille & $199(2,8 \%)$ & $22(2,7 \%)$ & $91(13,7 \%)$ & 6 & 4 & 12 \\
\hline Portefeuille Luc & $119(1,7 \%)$ & $10(1,2 \%)$ & $34(5,1 \%)$ & 4 & 2 & 7 \\
\hline Portefeuille Anatole & $206(2,9 \%)$ & $32(3,9 \%)$ & $21(3,2 \%)$ & 6 & 2 & 6 \\
\hline Portefeuille Martin & $1659(23,3 \%)$ & $57(7,0 \%)$ & $2(0,3 \%)$ & 6 & 2 & 2 \\
\hline Portefeuille Quentin & $327(4,6 \%)$ & $53(6,5 \%)$ & $85(12,8 \%)$ & 6 & 4 & 12 \\
\hline Total & $7129(82,8 \%)$ & $889(9,5 \%)$ & $662(7,7 \%)$ & $6(11,8 \%)$ & $5(9,8 \%)$ & $40(78,4 \%)$ \\
\hline
\end{tabular}

TABleau 2. - Proportion (nombre de pieds et de cultivars) des taros communs, intermédiaires et rares parmi les 56 bassins plantés par 9 foyers, soit 12 agriculteurs, car la famille d'Éric est composée d'Éric, le père, et de ses enfants non mariés, Arthur, Hervé et Bertrand. Ses enfants mariés, Pascal et Wendy, sont analysés comme foyer individuel.

La richesse en cultivars et la proportion de cultivars rares varie selon l'agriculteur (Tableaux 2 et 3); Bertrand, Wendy, Martin et Hervé sont les moins enclins à conserver des cultivars sans attraits majeurs. Toutefois, ces agriculteurs, à l'exception de Martin, plantent un nombre relativement homogène de pieds par cultivars (cf. Equitabilité de Tableau 3). Leurs cultivars sont soigneusement sélectionnés et multipliés. Mis à part Bertrand qui par son jeune âge, commence juste à accumuler des cultivars, le manque de temps et d'intérêt semble être la cause d'une telle sélection; Wendy, mariée dans un autre village dépourvu de tarodières irriguées, ne fait que des séjours très courts à Vētuboso ; Martin est un grand producteur de coprah et de kava qu'il revend dans un bar qu'il a aménagé dans la ville administrative de l'île, Sola. Hervé est un jeune homme qui ne porte que très peu d'intérêt aux tarodières et préfère les autres travaux domestiques. Ayant moins de cultivars à gérer suite à une sélection plus fine, ces quatre agriculteurs ont plus de temps et d'espace pour multiplier chacun des cultivars. Ce sont des « sélectionneurs » qui certes plantent peu de cultivars, mais qui par la multiplication des cultivars limitent les risques de perte d'un cultivar et donc de la diversité.

À l'inverse, Henri a un engouement incomparable pour la collection avec ses 46 cultivars. En conservant les cultivars comme des objets rares dans un musée, nous le qualifierons de « collectionneur ». En effet il ne jette jamais un cultivar même s'il ne correspond pas à ses attentes agronomiques ou gastronomiques. Sa stratégie lui a permis d'accumuler la plus grande variété de cultivars rares (35). Cependant, son équitabilité $(0,55)$ est inférieure à l'équitabilité de l'ensemble des portefeuilles $(0,6)$; il ne plante que très peu d'individus par cultivar rare, alors que $71 \%$ de son espace planté est occupé par seulement trois cultivars, marēwasalav, rov et lantar.

Entre les sélectionneurs qui répartissent au mieux la distribution de leur petit nombre de cultivars et les collectionneurs qui arborent les plus fortes richesses variétales, il existe un profil d'agriculteur qui maintien un nombre important de cultivars dans des proportions homogènes. Nous pouvons les qualifier de " conservateurs " car en maximisant la diversité et la richesse, ils conservent un nombre viable de cultivars protégés des intempéries du fait de leur duplication. Dans notre cas, Arthur serait le meilleur candidat sachant qu'il plante 27 cultivars (dont 17 rares) selon une équitabilité de 0,69.

Ainsi, malgré le riche portefeuille de cultivars plantés par unité familiale (19,7 en moyenne), seule une petite proportion de cultivars dits communs (6) occupe la plus grande partie de l'espace alors que la répartition hétéroclite entre les agriculteurs des cultivars rares (40) est à l'origine de la diversité du patrimoine à l'échelle du village. 


\begin{tabular}{|l|r|r|r|r|c|}
\cline { 2 - 6 } \multicolumn{1}{c|}{} & $\begin{array}{c}\text { Surface plantée: } \\
\mathrm{m}^{2}(\%)\end{array}$ & $\begin{array}{c}\text { Nombre de plants } \\
(\%)\end{array}$ & $\begin{array}{c}\text { Nombre } \\
\text { de cultivars }\end{array}$ & $\begin{array}{c}\text { Shanon-Wiener } \\
(\mathrm{H})\end{array}$ & $\begin{array}{c}\text { Equitabilité } \\
(\mathrm{E})\end{array}$ \\
\hline Eric & $730(16,9 \%)$ & $1448(16,8 \%)$ & 20 & 2,86 & 0,66 \\
\hline Arthur & $382(8,8 \%)$ & $805(9,3 \%)$ & 27 & 3,30 & 0,69 \\
\hline Hervé & $92,5(2,1 \%)$ & $192(2,2 \%)$ & 9 & 2,12 & 0,75 \\
\hline Bertrand & $2(0,4 \%)$ & $34(0,4 \%)$ & 6 & 2,22 & 0,86 \\
\hline Famille Eric & & & & & 0,67 \\
\hline Pascal & $1224(28,4 \%)$ & $2479(28,8 \%)$ & 27 & 3,19 & 0,79 \\
\hline Wendy & $218(5,1 \%)$ & $481(5,6 \%)$ & 14 & 3,01 & 0,88 \\
\hline Henri & $76(1,8 \%)$ & $81(0,9 \%)$ & 9 & 2,80 & 0,55 \\
\hline Achille & $1910(44,3 \%)$ & $2652(30,8 \%)$ & 46 & 3,05 & 0,73 \\
\hline Luc & $342(7,9 \%)$ & $312(3,6 \%)$ & 22 & 3,24 & 0,70 \\
\hline Anatole & $51(1,2 \%)$ & $163(1,9 \%)$ & 13 & 2,58 & 0,77 \\
\hline Martin & $96(2,2 \%)$ & $259(3,0 \%)$ & 14 & 2,95 & 0,62 \\
\hline Quentin & $189(4,4 \%)$ & $1718(19,9 \%)$ & 10 & 2,05 & 0,74 \\
\hline Total & $205(4,8 \%)$ & $465(5,4 \%)$ & 22 & 3,31 & 0,6 \\
\hline Moyenne par foyer & & & & & 3,41 \\
\hline Moyenne par bassin & 87,0 & $8610(100 \%)$ & 51 & 2,91 & 0,72 \\
\hline
\end{tabular}

Tableau 3. - Les portefeuilles de taros en chiffres des 9 foyers individuels soit, avec l'ensemble des membres de la famille Eric, des 12 agriculteurs : surface des bassins plantés, nombre de pieds de taro plantés, nombre de cultivars sélectionnés, fonction Shannon-Wiener (fondée sur la proportion de pieds des cultivars dans le portefeuille de chaque agriculteur) et équitabilité $\left(\mathrm{E}=\mathrm{H} / \mathrm{H}_{\max }\right)$. Les moyennes sont calculées sur la base de 9 foyers.

Cependant, en raison de la répartition hétérogène des cultivars rares entre les agriculteurs qualifiés de collectionneurs, de sélectionneurs ou de conservateurs, on peut noter que pour accéder à l'ensemble des cultivars d'un village, il faudrait visiter l'ensemble des bassins et donc étudier les comportements de l'ensemble des agriculteurs. En effet à l'intérieur des groupes d'agriculteurs que nous avons définis existe une importante diversité d'individus dont les choix diffèrent selon des objectifs plus intimes; leur portefeuille de cultivars est ainsi personnalisé.

\section{Pourquoi une telle diversité ?}

Face à l'hétérogénéité des portefeuilles de cultivars, nous devons nous interroger sur les raisons qui conduisent les agriculteurs à choisir un petit groupe de cultivars communs sursélectionnés (rov, marēwasalav, lantar, wasanto, vinmötöl et $w \bar{e} v \bar{e}$ ) en étudiant leurs performances, en particulier agronomiques et culinaires.

\section{Des cultivars performants}

Quatre des six cultivars communs sont reconnus pour leurs performances agronomiques et leur adaptation à un terroir. Marēwasalav repré- sente $19 \%$ des pieds plantés dans les 56 bassins observés. Ce cultivar est sélectionné pour sa rapidité de croissance, six mois au lieu de douze. Il est planté sur les pourtours intérieurs des bassins pour en faciliter la récolte plus précoce. De même wasanto $(10 \%)$, vinmötöl $(8 \%)$ et wēve $(6 \%)$ sont réputés pour leur corme de grande taille. Comme rov et lantar ne présentent aucune qualité culturale marquante alors qu'ils font parti des cultivars les plus plantés (respectivement 24 et $15 \%$ ), la performance agronomique des cultivars ne représente donc qu'une partie des critères de sélection.

Les multiples combinaisons recherchées, entre les cultivars, les modes et milieux de cultures, justifient la maintenance d'une richesse de cultivars aux formes, goûts et textures différents, judicieusement cuisinés selon de nombreuses recettes.

Des «bons» cultivars adaptés aux recettes quotidiennes

Les habitants de Vētuboso consomment chaque jour des taros : $12,8 \mathrm{~kg}$ de cormes secs par mois et par personne contre $3,2 \mathrm{~kg} \mathrm{de}$ riz $^{38}$. Malgré cette omniprésence alimentaire du taro,

38. Mesures par S. Caillon de la consommation de taros sur 16 jours dans un foyer de 7 personnes (septembre 2001) et mesures de celle du riz en relevant toutes les ventes sur un mois (juin 2003). 


\begin{tabular}{|l|c|c|c|c|c|c|}
\hline CULTIVARS & ROV & LANTAR & WĒVE & WASANTO & MARĒWASALAV & VINMÖTÖL \\
\hline Abondance (\%) & 24,0 & 15,1 & 6,0 & 10,1 & 19,2 & 8,4 \\
\hline Précocité & 2 & 2 & 2 & 2 & 1 & 2 \\
\hline Rendement & 4 & 4 & 3 & 1 & 2 & 3 \\
\hline Goût & 1 & 3 & 5 & 4 & 5 & 2 \\
\hline Texture & 1 & 4 & 4 & 3 & 5 & 2 \\
\hline Usage & \multicolumn{7}{|c|}{ Nalot Laplap } & Grillé \\
\hline
\end{tabular}

TABLEAU 4. - Classement des cultivars communs de Vētuboso selon leur abondance relative dans les 12 portefeuilles, leur précocité (1:6 mois ; $2: 1$ an), leur rendement ou taille du corme (1:plus gros), leur goût ou odeur (1:meilleur) et leur texture (du plus ferme : 1 au plus mou : 5).

ils n’ont assigné un usage culinaire particulier qu'à $25 \%$ des cultivars. Or, les six cultivars les plus plantés sont tous des taros aux propriétés organoleptiques spécifiques retenues pour une préparation particulière (Tableau 4). Ces propriétés sont dissociées en goût, en odeur et en texture. Plus un corme est ferme (tañañal $)^{39}$ et savoureux (dēdērēs), plus il est apprécié.

\section{Les cultivars à bouillir}

Les cormes de taro, classés parmi la nourriture (gengen) décrite comme « lourde $»^{40}$ sont le plus fréquemment consommés bouillis à l'eau et accompagnés de chou des îles cuits à l'eau ou au lait de coco (Cocos nucifera). Ils peuvent également être cuits dans le lait de coco (wōrqarqar) ou consommés avec de la chair de coco râpée (bigtöw). Tous les cultivars sont indifféremment bouillis même si certains, comme rov, sont préférés en raison de leur consistance ferme et de leur goût savoureux malgré une cuisson plus longue. Cependant les personnes âgées dépourvues de dents préfèrent les cultivars mous comme lantar, $w \bar{e} v \bar{e}$, wasanto et marēwasalav. S'ils sont bouillis trop longtemps, les cormes deviennent pâteux et sont décrits par le terme $\bar{m} \bar{e} d i t d a t$. Si les taros ont été récoltés trop tard ou pendant la saison rartan (avril-mai) et rartur (juin-juillet) (Caillon et Malau, 2002) ou s'ils ont été endommagés par un cyclone, ils seront dits görgör car contiendront trop d'eau.

\section{Les cultivars « à emporter »}

Lors des expéditions en forêt pour chasser le cochon sauvage ou lors des journées consacrées au travail dans les tarodières, les cormes sont grillés (tun), encore râpés pour être cuits dans des bambous (bōnësnës). Neufs cultivars présentent la particularité de n'être ni trop durs, ni trop mous et donc de rester tendres tout en cuisant vite, directement sur les braises : mako, marēwasalav, marēwasalav mamē, qiatgōl, siritimiat, titiritowetam, tortor, vinmötöl et wederebiliag. Seuls les cultivars les moins irritants et contenant un faible taux de matière sèche peuvent être cuits dans les bambous.

Afin de prolonger le temps de conservation des taros consommés lors de longs voyages, les cormes entiers non épluchés peuvent être cuits au four à pierres $(u m u)$. Les cormes cuits au four, au centre duquel de l'eau est ajoutée à l'aide d'un bambou, s'appellent qarnisis ${ }^{41}$. Tous les cultivars sont appréciés lorsque cuits selon cette méthode car elle estompe leurs différences organoleptiques, les cormes les plus mous devenant plus fermes.

\section{Les cultivars à nalot}

Plus occasionnellement, pour marquer certains jours, par exemple les rassemblements du samedi matin, l'arrivée de visiteurs ou encore une fête de famille, les cormes peuvent être transformés en nalot (löt) ou râpés en laplap.

Le nalot, dont les habitants des îles Banks passent pour être les spécialistes, est un pudding préparé, à Vanua Lava, principalement avec du taro. Les cormes sont d'abord cuits à l'étouffée ou bouillis à l'eau. Ils sont ensuite épluchés, avant d'être écrasés sur un grand plat en bois ovale $(t a b \bar{e})$ à l'aide d'un pilon en bois dur (vötulöt) spécialement réservé à cette préparation. Lorsque les cormes forment une pâte compacte et élastique, celle-ci est aplatie avec un morceau de l'épiderme d'une noix de coco. Le

39. Le terme « ferme » traduit à la fois la sensation de dureté et d'élasticité. Tañan̄al se différencie du terme meneg signifiant «dur».

40. Traduction du bislama. Les nourritures sont partagées en deux catégories : gengen (où gen signifie « manger ») désigne les féculents consommés quotidiennement et qui constituent la base de l'alimentation (taros, bananes, ignames, etc.), et bigbig qui désigne les accompagnements (chou, noix de coco, écrevisses, poissons, etc.)

41. Ce mode de cuisson "à la vapeur » est une spécialité des Banks. Les cormes peuvent être aussi cuits sans addition d'eau et seront dénommés weden. 
nalot est alors recouvert de nangailles $\bar{n} e$ (Canarium indicum) râpées vertes ou sèches, de velles wotag (Barringtonia edulis) ou plus communément de lait de coco.

Les villageois choisissent parmi quatorze types de nalot (Caillon et Malau, 2002) en fonction de leur goût, des aliments disponibles (noix de coco, nangailles et velles), du temps dont ils disposent et de la valeur donnée à certaines préparations. Par exemple le nalot nommé wirmamēgin est préparé pour "assurer une bonne croissance des enfants $»^{42}$ car il est moins gras que les autres, le lait de coco cru étant mélangé à de l'eau.

Un bon nalot est un pudding élastique fait avec des cultivars fermes dit tañañal, à fort taux de matière sèche, tels que les deux cultivars rov et lōkreg. Pour faciliter le malaxage de la pâte, ils sont généralement mélangés à d'autres cultivars plus tendres et savoureux en particulier lantar. Rov est tout de même préféré à lōkreg car en plus d'une texture adéquate, son odeur et sa saveur plaisent particulièrement aux amateurs de nalot. Les taros cultivés en tarodières irriguées sont préférentiellement utilisés pour le nalot car leur texture est plus ferme.

\section{Les cultivars à laplap}

Le laplap, un autre plat courant aux îles Banks, est cuisiné à partir d'une plus grande diversité d'espèces comme le manioc, les ignames (dëm, Dioscorea alata; tamag, Dioscorea esculenta; bōōr, Dioscorea nummularia), les bananes et plus occasionnellement les taros. Pour préparer un laplap de taro, les cormes sont râpés crus, puis empaquetés dans des feuilles à laplap (Heliconia indica) et cuits dans un four à pierres. À Vanua Lava, le laplap n'a pas la même place que dans les autres îles du Vanuatu et du groupe des Banks, car les femmes préfèrent utiliser les taros en nalot, plus valorisé. Afin d'éviter les irritations ou démangeaisons causées par les cristaux d'oxalate de calcium, des cultivars mous mölumlum et à haute teneur en eau sont recherchés pour le laplap. Dix cultivars (lantar malgias, lantar lamkör, relenman, sarēe, sestañ, suwbē, wakata mamē, wamal, wasanto, wasanto mamē, $w \bar{e} \bar{e}$ et wotliev) remplissent ces conditions, en particulier s'ils sont cultivés en immersion simple dans les rivières aménagées. Ils sont alors moins riches en matière sèche, sont râpés plus facilement et sont moins irritants.

Ainsi, les cormes des six cultivars communs répondent de par leur goût et leur texture aux multiples exigences que requiert chaque recette de cuisine. Tous sont particulièrement appréciés lors de leur consommation quotidienne : rov est le cultivar à bouillir et à nalot, lantar, wēve et wasanto sont râpés en laplap, et marēwasalav et vinmötöl sont appréciés grillés.

\section{Les cultivars et leur préparation}

lors des fêtes et des cérémonies

\section{Les fêtes du village}

Les cormes cuits au four et préparés sous forme de nalot sont indispensables à tous les grands rassemblements. Lorsqu'une famille célèbre un événement important, elle invite des parents et amis, qui apportent des cormes crus ou cuits à ceux qui reçoivent. Cependant, les invités ne peuvent pas offrir n'importe quel cultivar. Par exemple, marēwasalav, dont le corme atteint en six mois la taille à laquelle parviennent les autres cultivars en un an, doit être néanmoins récolté après dix mois pour que le corme ait un «vrai» goût de taro et «non d'igname $»^{43}$. Si un invité l'apporte à une fête, l'hôte pourra suspecter que le taro n'est pas complètement mûr car sa taille ne sera pas révélatrice. Etant facile à cultiver, il est probable que l'hôte proposera déjà en quantité ce cultivar, même s'il n'a pas une grande qualité gustative. Les invités se doivent donc d'apporter des cultivars plus rares ou meilleurs, c'est-à-dire plus savoureux et fermes.

Pour les événements importants, certains nalot sont plus valorisés que d'autres. Celui qui est nommé lötnemereñ, à base de taros et de nangailles sèches, est incontournable lors des fêtes. D'autres types de nalot, choisis en fonction du goût et des habitudes familiales, peuvent être ajoutés. Les plus répandus sont lötsalsim et salō$q \bar{o} s$, recouverts de lait de coco respectivement roussi et crémeux, le premier étant plus long de cuisson que le deuxième. Un bon nalot sera d'autant plus apprécié qu'il est préparé avec le cultivar rov qui lui donnera son élasticité et du cultivar lantar, de texture plus molle mais réputé pour son goût.

\section{Les cérémonies de naissance}

Lorsqu'une femme est enceinte pour la première fois, sa famille et celle de son époux organisent, au terme de six mois de grossesse, une

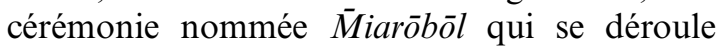
autour d'un repas constitué de taros cuits dans le four à pierres et de nalot, en particulier lötneme- 
$r e \bar{n}$. À cette cérémonie sont conviés des parents, en premier lieu les frères, sœurs et oncles maternels de la femme, ainsi que des parents éloignés.

Dans les temps anciens, les femmes accouchaient dans leur maison, avec l'aide d'une sagefemme. Speiser $(1996: 248)$ relève que dès la naissance de l'enfant, la sage-femme enfonçait un doigt dans la gorge du nouveau-né, pour vérifier qu'il avait deux entrées, une pour l'eau et une pour les aliments solides. Elle lui donnait alors de l'eau de coco et lui bourrait la bouche avec du nalot de taro, marquant ainsi son entrée dans le monde des hommes.

De nos jours, lors de la cérémonie de la première dent, Gövutlēw, le bébé doit ingérer un nalot lötnemereñ rasqiat préparé, à la différence des nalot pour adultes, avec des cultivars de texture molle (cf. cultivars à laplap) et recouvert de nangailles sèches. Il est dénommé rasqiat car les cormes râpés sont cuits comme un laplap puis pilonnés sur un plateau en bois pour en assouplir la texture selon les procédés utilisés pour le nalot. Ce plat traditionnel est aujourd'hui le premier aliment solide ingéré par un jeune enfant et la commensalité à cette occasion est le premier acte social notable de sa vie marquant son entré dans le jeu des relations d'échange. La préparation particulière de ce nalot signifie, outre le fait que le jeune enfant peut difficilement mâcher, qu'il n'est pas encore un être humain complet et que la constitution de sa personne se fera peu à peu à travers la construction de relations avec d'autres humains et non-humains comme les esprits et les ancêtres.

\section{Les cérémonies de mariage}

La présence de nalot est également indispensable au mariage, qui suit traditionnellement un long processus au cours duquel la famille du garçon offre des présents à la famille de la fille, notamment des plantes pérennes ${ }^{44}$ à cultiver offertes pour marquer l'accord et pour symboliser la durée. Lors de la cérémonie clôturant le mariage, Dalagè, d'autres plantes sont offertes par l'époux pour être cultivées (taros avec leur partie aérienne, noix de coco germées, rejets de bananiers, choux, canne à sucre,...) ou consommées (un ou deux nalot lötnemerēn, noix de coco immatures, bananes vertes), accompagnées de paniers et de 40 à 45000 vatus $^{45}$, enroulés sur des tiges de roseau traditionnellement plantées au milieu des cadeaux. La future épouse peut en retour donner un peu de monnaie, par exemple 2000 vatus, pour avoir des droits dans la maison de son mari mais aussi pour que sa famille conserve une certaine autorité sur sa personne en cas de litiges conjugaux.

\section{Les cérémonies lors d'un décès}

Lors des funérailles, diverses cérémonies ont lieu : mise en terre, cérémonie Ununseg pour le partage des terres, distribution du collier d'hibiscus qui instaure les tabous alimentaires (en particulier le taro pour la famille proche), repas communautaires et destruction des biens du défunt lors de la cérémonie Vono $\bar{m} \bar{o} l \bar{o}$ signifiant « espace ouvert». Plus le mort est respecté, plus la destruction de taros, de cocotiers ou de bétail sera intense. Le $5^{\text {ème }}$ jour, le premier grand repas consistait selon Codrington (1891: 271) à «manger la tombe »; le $10^{\text {ème }}$ jour, un autre repas permettait de "manger les jours ». Les habitants de Vētuboso servent aujourd'hui préférentiellement le nalot lötnemere $\bar{n}$ (taros et nangailles) mais il est possible qu'il existait dans les temps anciens, comme dans d'autres îles du groupe des Banks, un interdit sur la consommation des noix de coco levé dix jours après le décès. Le nalot institué pour ce repas du dixième jour était alors certainement composé de taros et de noix de coco. Ce repas est suivi, tous les 5 ou 10 jours, de repas réunissant plus ou moins de monde selon la notoriété et la réputation du défunt. Ces repas sont clôturés au centième ou millième jour, par un partage de notamment 1000 à 5000 cormes de taro cuits au four. Aucun cultivar particulier n'est recommandé pour cette cérémonie.

\section{Les cérémonies statutaires}

Dans les temps anciens, lorsque les hommes étaient encore impliqués dans les institutions statutaires comme la hiérarchie de grades soq et les sociétés secrètes timiat, deux cultivars nommés tortor et mako étaient réservés à ces institutions ${ }^{46}$. Ces cultivars étaient grillés par celui ou ceux qui étaient reclus dans la maison des hommes de la hiérarchie du $s o q$ pour acquérir un rang.

44. Lorsque la jeune-fille est trop jeune pour être mariée, la famille de son futur époux la réserve au cours de la cérémonie Gonwōmōtō. À cette occasion, deux fruits germés de cocotier sont plantés côte à côte attachés ensemble par leur bourre. Les deux cocotiers symbolisent la solidité du contrat entre les deux familles.

45. En juin 2002, 1 euro = 129 vatus. Dans les temps anciens, la monnaie de coquillage était utilisée.

46. La hiérarchie $s o q$ a disparu depuis le milieu du $\mathrm{XX}^{\mathrm{e}}$ siècle. Certains chefs coutumiers cherchent aujourd'hui à la faire revivre. Les sociétés secrètes existent toujours même si leur statut a un peu changé. Les hommes y acquièrent des masques timiat qui représentent les ancêtres, également nommés timiat. 
Quand la période de réclusion s'achevait, l'initié préparait un nalot sur un plat en bois de petite taille, avec un pilon plus petit que la normale, qu'il plaçait sur ses mains croisées en chantant. Il marquait trois temps d'arrêt pendant ce chant avant de pouvoir porter un morceau de nalot à sa bouche; l'ingestion marquait son ascension dans la hiérarchie soq.

Le nalot devait être coupé en forme de losange et non en rectangle comme pour les repas quotidiens ou festifs. Cette forme réservée aux gradés évoque un masque des sociétés secrètes timiat, nommé $v \bar{e} v$. Le couteau en bois, meteges, utilisé pour tailler ces formes dans le pudding, est sculpté de motifs réservés aux gradés. Dix figures, représentant les dix grades du soq, étaient autrefois matérialisés par dix fours à pierres ou feux tabous situés dans la maison des hommes, et de nos jours encore, seuls les hommes peuvent toucher un objet sculpté.

Le type de nalot ingéré à cette occasion est controversé. Selon les chefs coutumiers actuels, il s'agit d'un nalot lötnemereñ à base de taros cuits au four ou bouillis et de nangailles séchées, alors que pour un informateur plus âgé, ayant connu cette institution, il s'agit d'un nalot dénommé wagaretow, interdit aux femmes et préparé avec des taros grillés surmontés de velles séchées. Si le premier nalot est élastique car préparé avec des cormes fermes comme rov, le second fait avec des cormes tendres ne contenant pas trop d'eau, a une consistance molle. Cette ingestion de cormes mous, comme pour les jeunes enfants ou les personnes âgées, témoigne de l'affaiblissement de l'homme qui acquiert le grade. On peut souligner que les cultivars à rôtir appropriés pour le nalot wagaretow tels que qiatgōl, mako, tortor, vinmötöl et wederebiliag, sont tous d'anciens cultivars présents dans les récits coutumiers soit parce qu'il ont été apportés par un héros mythique (Wōmodō ou Biliag), soit parce qu'ils sont reconnus dans l'ensemble du village comme les « premiers taros de l'île». Les taros grillés avaient une importance sociale aujourd'hui perdue; ils sont maintenant uniquement consommés comme nourriture d'appoint.

À l'inverse, la cuisson en marmite, permettant une cuisson plus rapide des cormes que le four ou les braises, est aujourd'hui la plus pratiquée pour une cuisine journalière mais aussi pour la cuisson des cultivars à nalot alors qu'auparavant les cormes étaient cuits au four à pierres ou grillés comme pour le nalot wagaretow. L'intégration de cette technologie exogène a engendré la création d'un nouveau besoin en cultivars fermes que rov a comblé au mieux comme le montre son omni- présence dans les jardins. En effet le cultivar rov n'a été « découvert » que très récemment par un agriculteur du même nom il y a une ou deux générations. Dans un contexte de changements environnementaux et sociaux, l'évolution des critères de préférence s'accompagne de processus de sélection variétale efficaces, permettant une innovation continuelle.

Pour acquérir dans les temps anciens les grades les plus élevés, une grande quantité de monnaie de coquillage était nécessaire. Les hauts gradés pouvaient chercher à s'en approvisionner à l'extérieur de l'île, en particulier en provenance du banc de sable Rowa au nord-ouest de Vanua Lava. L'acquisition de monnaie à l'extérieur du groupe social était fondamentale pour les hommes de Vētuboso vivant à l'ouest de l'île le long de plages volcaniques sans récifs où il n'y avait pas de coquillages. Mais il leur fallait, en retour, proposer des biens de valeur, dont faisaient partie les taros, d'une qualité exceptionnelle, ainsi que les porcs. Aujourd'hui l'institution du soq n'existe plus, les sociétés timiat n'ont plus l'importance qu'elles avaient dans les temps anciens et les relations d'échanges entre les îles se sont relâchées. Cependant, les taros de Vanua Lava sont toujours réputés et ils sont aujourd'hui obtenus par troc ou par achat avec de la monnaie nationale. Par exemple lorsque les habitants de Mota Lava organisent de grandes fêtes nécessitant une nourriture de prestige, ils acquièrent des taros entiers auprès des habitants du village de l'est de Vanua Lava, Lalgêtak, soit en les achetant, soit en les échangeant contre des produits de la mer. Les taros de Lalgêtak étant plantés en rivières aménagées, les habitants de Mota Lava consomment par obligation des cormes mous.

Ainsi, la recherche de certains cultivars pour les fêtes et les cérémonies, et la préservation de ceux utilisés dans les temps anciens par les hommes souhaitant progresser dans la hiérarchie de grades soq montrent que le choix des cultivars ne relève pas simplement de propriétés agronomiques ou organoleptiques, mais aussi de leur valeur sociale liée à leur origine ou leur mode de culture. De plus, la vie sociale débute et se termine par l'ingestion, par l'intéressé ou par ses proches, du plat culturellement le plus valorisé, le nalot de taros. Le taro cuit au four ou préparé en nalot est ainsi nécessaire à chaque moment important de la vie sociale. Une bonne récolte de taros permet d'honorer ses devoirs et de participer aux fêtes en offrant les «bons » cormes en quantité importante. Ces prestations sociales montrent qu'un homme et sa famille sont des gens de valeur, dotés de connaissances et de res- 
pect indispensables pour réussir la culture du taro. Toutefois, un homme occupé par d'autres activités peut aujourd'hui acquérir des cultivars de valeur auprès d'un cultivateur qui acceptera de lui vendre une parcelle de taros sur pieds ou éventuellement quelques plants.

\section{Des cultivars 'sociaux'}

Nous avons vu que la majorité des cultivars communs, peu nombreux mais plantés sur de grandes surfaces, doivent leur popularité à des atouts agronomiques ou organoleptiques, pour leur utilisation dans des recettes quotidiennes ou au cours de cérémonies. Or 40 des 51 cultivars de notre échantillon de 12 agriculteurs sont peu multipliés et 45 cultivars des 96 inventoriés dans le village ne sont pas présents. Au sein du patrimoine villageois, 85 cultivars, soit plus de $88 \%$, peuvent être considérés comme rares. D'après les dires des agriculteurs, ils ne sont pas particulièrement performants ou plaisants à consommer. Il est donc essentiel de comprendre pourquoi ces cultivars sont conservés.

Les agriculteurs plantent un faible nombre de pieds par cultivar venant d'autres îles (17 cultivars introduits représentent $25 \%$ des pieds de cultivars rares) car, lorsque leur introduction est récente ; ils sont d'abord testés avant d'être éventuellement multipliés. Si ces cultivars introduits ne présentent pas de qualité intéressante, ils sont tout de même conservés en un petit nombre d'exemplaires pour leur connotation exotique.

Si un homme (32 noms) ou, plus rarement, une femme (cinq anciens noms : regeltëv, rēgēt, rēelègtēl, rēwurweg et römöwuler) donne son nom à un nouveau cultivar qu'il ou elle a découvert dans un bassin en jachère $(25 \%$ des pieds de cultivars rares), ses descendants le conserveront comme un héritage. Au Vanuatu, on ne parle pas de droit de propriété de la terre, mais de droit d'usage. Chaque personne s'approprie ce qu'elle plante et non le sol qui nourrit ses cultures. Le nouveau cultivar repéré est approprié par son découvreur et porte ainsi son sceau. Son souvenir à travers son nom porté par un cultivar traversera le temps sous la forme d'un organisme vivant, le taro.

Les agriculteurs aiment à préserver les cultivars (10 cultivars représentant $22,5 \%$ des pieds de cultivars rares) mentionnés dans les mythes fondateurs (tañevsos, bulalef, burmatan, qiatgōl, qiatqet) et les récits dits coutumiers ( $\bar{m} \bar{o} v \bar{v} l$, rēgēt, wasē, wemenriver, wederebiliag). Dans cette société à tradition orale, le taro est l'illustration vivante de l'histoire qu'un père conte avec fierté à ses enfants par exemple à l'occasion de la ren- contre avec ce cultivar « historique " lors des travaux dans les bassins irrigués.

$\mathrm{Si}$ le cultivar arbore une forme insolite (8 cultivars comptant pour $12,5 \%$ des pieds), l'agriculteur le gardera également comme une signature qui différenciera ses bassins de ceux des autres agriculteurs. C'est le cas de cultivars dont les pétioles ((bus)ōr, master et nalumlum), les cormes ((we)menriver, m̄èvinvian, siritimiat et wotmēlèv) et les feuilles (taltal) présentent des couleurs inhabituelles.

Un seul cultivar, aujourd'hui oubliés par une grande partie des habitants du village, avait autrefois une valeur magique. Lorsqu'un homme pratiquant la « magie noire » empoisonnait un autre, ce dernier devait avaler un corme cru du cultivar nommé dogon pour que sa famille connaisse ses chances de survie : s'il l'avalait sans le rejeter, il survivrait à cette agression; dans le cas contraire, il succomberait au poison.

Lorsqu'un agriculteur trouve ou introduit un nouveau cultivar, il le plantera, le multipliera et le distribuera avec soin. L'ampleur de la dispersion conditionnera la renommée du découvreurdistributeur, de son vivant et après sa mort. Lorsque le cultivar n'a pas de qualités exceptionnelles, son aire de distribution dépendra d'autant plus de la volonté avec laquelle le « propriétaire » le dispersera par dons à travers ses réseaux sociaux. Enfin, si un agriculteur cherche à obtenir un cultivar particulier planté par un autre membre de la communauté, il devra soigner ses relations sociales pour l'acquérir moyennant un contre-don non immédiat et n'étant pas forcément de même nature. Si en théorie on peut demander un cultivar à n'importe quel habitant de l'île, en pratique les dons se cantonnent aux personnes proches, c'està-dire aux membres du groupe matrilinéaire restreint et aux personnes en relation de mariage ou d'adoption. Quand un cultivar est fortement apprécié, le réseau de distribution peut s'élargir à toute la communauté, voire à l'île, parfois par le biais du vol.

Enfin mais surtout, les communautés villageoises façonnent leur identité sociale ou leur « badge d'identification » (Laycock, 1982) par les connaissances du milieu qu'elles arriveront à acquérir de manière empirique. De ce degré de connaissance dépendra la richesse des savoirfaire et donc la qualité de produits originaux. Dans le cas des plantes cultivées et en particulier du taro à Vētuboso, l'unicité du matériel végétal, soit des cultivars, existant de manière exclusive à l'intérieur d'une société (entre les personnes) et entre des sociétés, crée un intérêt pour l'échange. Les réseaux sociaux organisent ces échanges qui 
constituent la base fondamentale à l'évolution de toutes sociétés. Comme le souligne Bonnemaison (1991), cette "abondance de produits rares ", ici de taros, génère une "générosité sociale » consolidant les relations entre les personnes à travers le tissage d'un réseau d'échange complexe.

Ainsi, la majorité des cultivars sont conservés non pas pour leurs seuls usages culinaires mais aussi pour leur valeur sociale. N'étant pas choisis pour leurs qualités gastronomiques ou agronomiques, les cultivars rares sont conservés en petit nombre en tant que curiosité singulière, qu'élément magique, que support de mémoire d'un voyage passé, d'un héritage familiale ou culturel, et comme un moyen d'échange. L'importance d'une justification sociale de la diversité prend une valeur essentielle dans un contexte où les produits de l'agriculture n'ont pas seulement une utilité alimentaire mais acquièrent également une finalité sociale comme l'a déjà justement souligné Bonnemaison (1991) pour d'autres îles de l'archipel.

\section{Conclusion : \\ leçons pour une stratégie de conservation in situ}

Les habitants de Vanua Lava sont reconnus pour la qualité et l'unicité de leurs taros cultivés dans des tarodières irriguées aux pratiques uniques au Vanuatu. Cette différence est " cultivée » dans le but de mieux échanger à travers des réseaux de parenté omniprésents. Ce besoin d'identification par la singularité existe aussi à l'échelle de la personne. Ainsi, chaque portefeuille de cultivar est composé selon des motivations personnelles. Cette répartition inégale des pieds de taros entre les cultivars et des cultivars entre les agriculteurs, nous a amené à analyser les différences de stratégies de gestion de la diversité entre les agriculteurs; le sélectionneur a ainsi pu être différencié du collectionneur. Le sélectionneur choisit un petit nombre de cultivars dont chacun sera planté dans des proportions homogènes tandis que le collectionneur conserve un maximum de cultivars sans prendre la peine de multiplier ceux qui ne répondent pas à ses attentes agronomiques ou organoleptiques.

À Vanua Lava, les conditions socio-environnementales sont optimales pour observer une forte diversité intra-spécifique: l'écologie
« humide » est propice à la culture du taro dans des jardins proches des habitations, l'île est en dehors des grands circuits commerciaux mais reste au carrefour des échanges traditionnels, les habitants ont une volonté très marquée de retour à la « coutume », et les enfants quittent rarement le village pour une éducation secondaire. Cependant, la perte de diversité variétale du taro est d'actualité sur d'autres îles des Banks (Mota, Gaua et Mota Lava) ou dans le reste de l'archipel (entre autres Pentecôte, Ambae et Tanna) où le principal facteur d'érosion est la compétition inter-spécifique avec des espèces introduites plus tolérantes à la sécheresse, comme le manioc et la patate douce (Ipomoea batatas) que les agriculteurs peuvent planter plus près de leurs habitations (Caillon et al., 2004).

La conservation des cultivars de taro au Vanuatu est donc un problème actuel d'autant plus qu'une maladie (Phytophthora colocasiae), responsable de la destruction du patrimoine génétique de Samoa, cerne l'archipel. La conservation $^{47}$ de la diversité du taro ne doit pas être envisagée comme la création d'un musée villageois même si la collection était déterminée et surveillée par un groupe de gardiens agriculteurs. En effet, le patrimoine des taros du village ne s'exprime sous sa forme holistique que si l'ensemble des portefeuilles des agriculteurs, en particulier les cultivars rares, est pris en compte. De plus, la composition de ces portefeuilles évolue en fonction des besoins comme l'a souligné l'exemple de la disparition des cultivars à griller au profit des cultivars à bouillir. Une telle diversité ne trouve son fondement que dans la multiplicité des stratégies d'acquisition-sélectionconservation dynamiques des cultivars propres à chaque habitant. Dans les méthodes de conservation in situ, on fait souvent référence à des personnes ressources (Maxted et al., 2002). Doit-on confier une telle responsabilité au collectionneur dont on a montré le manque de durabilité de son portefeuille ? Plus qu'une personne ressource, il faudrait parler d'une unité villageoise pour conserver l'ensemble du patrimoine. De plus, l'agrobiodiversité, plus qu'une collection de variétés cultivées, est un ensemble de processus allant de la création (fécondation, mutation) à la diffusion en passant par la sélection et la multiplication. De ces processus, les aspects sociaux apparaissent fondamentaux. La question se pose alors de savoir s'il est possible

47. La conservation in situ des plantes cultivées est fondée sur la gestion locale de l'agrobiodiversité. Les conservateurs ne cherchent pas à maintenir des génotypes mais des mécanismes dynamiques à travers lesquels la plante s'adapte aux pressions environnementales et sociales qui lui ont permis de développer ses propriétés distinctives. La conservation in situ est une méthode complémentaire de la conservation ex situ dont le but est de conserver des gènes d'espèces cibles retirées de leur habitat naturel (banques de gènes, collections vivantes). 
de préserver les processus sans préserver les dynamiques sociales et culturelles.

Les collections ex situ répondent également à un principe d'exhaustivité ; ces collections vivantes de plantes dissociées de leur milieu naturel, et regroupées dans un espace commun et contrôlé (station scientifique), se doivent de regrouper et de rassembler un maximum de diversité dans un espace restreint. Ces collections étant difficilement maintenues face aux intempéries climatiques et aux bouleversements sociaux, des collections noyaux sont créées avec un nombre plus restreint de variétés, donc plus facile à gérer. Ces collections « miniatures » sont sélectionnées pour représenter au mieux la diversité des génotypes présents dans la collection d'ensemble en choisissant les individus les plus différents génétiquement, morphologiquement et/ou géographiquement.

En milieu villageois, multiplier les acteurs participant à la conservation augmente les risques de perte car le responsable d'un projet de conservation maîtrisera moins l'environnement social s'il doit dialoguer avec un grand nombre d'agriculteurs ; le plan de conservation in situ s'alourdit alors que sa force réside dans sa souplesse. Le principe de collection noyaux in situ serait donc une solution pour un maintien sécurisé de l'agrobiodiversité dans un village. Dans ce cas, le collectionneur doit s'associer à un conservateur, plus jeune, afin de lui transférer des cultivars rares que le conservateur multipliera progressivement. Les rapports entre la richesse en cultivars et l'équitabilité seraient ainsi maximisés.

Cette étude sur une forme de gestion conservatoire de la diversité des taros est, depuis juillet 2004, au cœur d'un projet sur « la préservation et l'utilisation de l'agrobiodiversité des plantes à racines et tubercules » réalisé par le Ministère de l'Agriculture du Vanuatu. Une telle approche multidisciplinaire est à recommander lorsque le sujet d'étude porte sur les relations entre les êtres humains et leur environnement.

\section{Remerciements}

Entre 2001 à 2003, ce projet a été financé par la Région Centre, le CIRAD (Centre International de Recherche Agronomique pour le Développement) et l'IRD (Institut de Recherche pour le Développement). Ce travail n'aurait pu être réalisé sans l'aide généreuse des agriculteurs de Vētuboso. Les connaissances des Chefs coutumiers Eli Field Malau et Hosea Woras ont été particulièrement fructueuses. Nous tenons à remercier avec insistance Sabine Hess et
Catriona Hyslop pour avoir complété certains écueils ainsi que Jean-Paul Lescure et Dominique Guillaud pour leur relecture motivée et rigoureuse.

\section{BIBLIOGRAPHIE}

BARrAU Jacques, 1983. Les Hommes et leurs aliments. Esquisse d'une histoire écologique et ethnologique de l'alimentation humaine, Paris, Messidor/Temps Actuels.

BonNEMAISON Joël, 1991. Le taro-roi : une horticulture d'abondance dans l'Archipel du Vanuatu (Mélanésie), in R. Blanadet, J.M. Amat-Roze, S. GuichardAnguis, C. Balaize et A. Louchet (éds), Aspects $d u$ monde tropical et asiatique : hommage à Jean Delvert, Paris, Presses de l'université de ParisSorbonne, pp. 305-315

Caillon Sophie and Eli Field Malau, 2002. Coconuts and taros from the West Coast of Vanua Lava (Vanuatu): an ethno-agronomic inventory, Orléans, IRD.

Caillon Sophie, José Quero-García and Luigi GuARINO, 2004. Taros in Vanuatu: toward a dynamic conservation strategy, LEISA 20, 1, pp. 18-20.

Caillon Sophie, José Quero-García, Jean-Paul LesCURE and Vincent LEBot, 2005 (sous presse). Nature of taro (Colocasia esculenta (L.) Schott) genetic diversity prevalent in a Pacific oceanic island, Vanua Lava, Vanuatu, Genetic Resources and Crop Evolution.

Codrington Robert H., 1969 [1891]. The Melanesians, Studies in their anthropology and folklore, Oxford, Clarendon Press.

EsCOBAR Arturo, 1998. Whose knowledge, whose nature? Biodiversity, conservation, and the political ecology of social mouvements, Journal of Political Ecology 5, pp. 53-82.

Haudricourt André Georges, 1964. Nature et culture dans la civilisation de l'igname : l'origine des clones et des clans, L'Homme 4, 1, pp. 93-104.

Hyslop Catriona, 1999. Diksonari blong vurës lanwis, Wes Vanua Lava, Vanuatu, Port Vila, Centre culturel du Vanuatu.

IVANCIC Anton and Vincent LEBOT, 2000. The genetics and breeding of taro, Montpellier, CIRAD.

Jolly Margaret, 1994. Women of the Place. Kastom, Colonialism and gender in Vanuatu, Chur (Switzerland), Harwood Academic Publishers GmbH.

KaHN Miriam, 1984. Taro irrigation: A descriptive account from Wamira, Papua New Guinea, Oceania 54, 3, pp. 204-222.

KIRCH Patrick V., 1994. The wet and the dry. Irrigation and agricultural intensification in Polynesia, Chicago-London, The University of Chicago Press. 
KREBS Charles, 1994. Ecology: the experimental analysis of distribution and abundance, New York, Harper Collin College Publishers.

LANOUGUÈRE-BRUNEAU Virginie, 1999. Les tarodières irriguées de l'île Vanua Lava, une marque identitaire dans le système social inter-îles (îles BanksVanuatu), JATBA 41, pp. 61-91.

LAYCOCK D. C., 1982. Melanesian linguistic diversity: a melanesian choice?, in R. J. May et H. Nelson (eds), Melanesia: beyond diversity, Canberra, Australian National University, Research School of Pacific Studies, pp. 33-38.

MaXted Nigel, Luigi Guarino, Landon Myer and Edwin A. Chiwona , 2002. Towards a methodology for on-farm conservation of plant genetic resources, Genetic Resource and Crop Evolution 49, 1, pp. 3146.

Quantin Paul, 1982. Vanuatu. Agronomic potential and land use map. Explanatory Notes, Paris, ORSTOM Éditions.

Shiva Vandana, 1996 (june). Agricultural biodiversity, intellectual property rights, and farmers' rights. Economic and political weekly, pp. 1621-1631.

SPEISER Felix, 1996 [1923]. Ethnology of Vanuatu, an early twentieth century study, Bathurst, Crawford House Publishing.

SpRIGGS Matthew. J., 1981. Vegetable kingdoms: Taro irrigation and Pacific prehistory, unpublished $\mathrm{PhD}$ thesis, Canberra, Australian National University.

SpRIGGS Matthew. J., 1982. Irrigation in Melanesia: Formative adaptation and intensification, in R. J.
May and N. Hank. (eds), Melanesia beyond diversity, Camberra, Australian National University, Research School of Pacific Studies, pp. 309-324.

Vargo Agnes et Lisa Ferentinos, 1991. A rapid rural appraisal of taro production systems in Micronesia, Hawai'i and American Samoa, Honolulu, University of Hawai'i, Pacific Agricultural Development Office.

VIENNE Bernard, 1984. Gens de Motlav. Idéologie et pratique sociale en Mélanésie, Paris, Société des Océanistes, Publications de la Société des Océanistes 42 .

VIENNE Bernard (ms). La nomenclature des clones de taro qeta-Colocasia esculenta (L.) Schott - Contribution à l'ethnobotanique de l'archipel des îles Banks - Nouvelles-Hébrides, ORSTOM.

Walter Annie et Fabienne Tzerikiantz, 1999. La tarodière irriguée : un système d'agriculture diversifié, JATBA 41, 2, pp. 185-219.

WeLls Nancy, 2000. The 1999 Vanuatu national population and housing census, Port Vila, Vanuatu National Statistics Office.

YEN Douglas E., 1990. Environment, agriculture and the colonisation of the Pacific, in D.E. Yen and M.J. Mummery (eds), Pacific Production systems: approaches to economic prehistory, Canberra, Department of Prehistory, Research School of Pacific Studies, Australian National University, Occasional papers in Prehistory 18, pp. 258-277. 